CERN-PH-TH/2005-039

hep-th/0503123

\title{
D-Brane Boundary States in the Pure Spinor Superstring
}

\author{
Ricardo Schiappa ${ }^{\dagger, \ddagger}$ and Niclas Wyllard ${ }^{\S}$ \\ †CAMGSD, Departamento de Matemática, Instituto Superior Técnico, \\ Av. Rovisco Pais 1, 1049-001 Lisboa, Portugal \\ ${ }^{\ddagger}$ Faculdade de Engenharia, Universidade Católica Portuguesa, \\ Estrada de Talaíde, 2635-631 Rio de Mouro, Lisboa, Portugal \\ $\S$ Department of Physics, Theory Division, CERN, \\ 1211 Geneva 23, Switzerland \\ schiappa@math.ist.utl.pt, wyllard@cern.ch
}

\begin{abstract}
We study the construction of D-brane boundary states in the pure spinor formalism for the quantisation of the superstring. This is achieved both via a direct analysis of the definition of D-brane boundary states in the pure spinor conformal field theory, as well as via comparison between standard RNS and pure spinor descriptions of the superstring. Regarding the map between RNS and pure spinor formulations of the superstring, we shed new light on the tree level zero mode saturation rule. Within the pure spinor formalism we propose an explicit expression for the D-brane boundary state in a flat spacetime background. While the non-zero mode sector mostly follows from a simple understanding of the pure spinor conformal field theory, the zero mode sector requires a deeper analysis which is one of the main points in this work. With the construction of the boundary states at hand, we give a prescription for calculating scattering amplitudes in the presence of a D-brane. Finally, we also briefly discuss the coupling to the world-volume gauge field and show that the D-brane low-energy effective action is correctly reproduced.
\end{abstract}




\section{Contents}

$1 \quad$ Introduction and Summary 2

2 Pure Spinors and the Covariant Superstring 3

2.1 Tvpe II Covariant Superstrings in a Flat Background . . . . . . . . . 4

2.2 Berkovits' Tree Level Zero Mode Saturation Rule from RNS . . . . . 12

2.3 Mode Expansions . . . . . . . . . . . . . . . . . . . . . 16

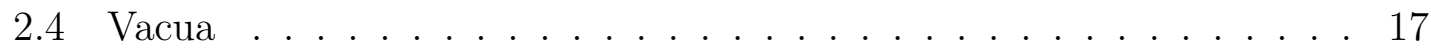

3 Pure Spinor Boundarv States in Flat Spacetime 18

3.1 The Open String Picture: Sigma Model Boundarv Conditions . . . . 19

3.2 The Closed String Picture: D-Brane Boundarv States . . . . . . . . . 20

3.3 Boundarv State: Non-Zero Mode Sector . . . . . . . . . . . . . . 21

3.4 Boundarv State: Zero Mode Sector . . . . . . . . . . . . . . . . . 22

3.5 The Generalisation to Lower Dimensional D $p$-Branes . . . . . . . . 26

\begin{tabular}{|ll}
4 Applications and Checks & 28
\end{tabular}

4.1 Calculating Scattering Amplitudes . . . . . . . . . . . . . 28

4.2 Coupling to a Gauge Field . . . . . . . . . . . . . . 31

5 Final Remarks 36

\begin{tabular}{|l|l}
\hline A Flat $\mathcal{N}=1, d=10$ Superspace & $\mathbf{3 7}$
\end{tabular}

\begin{tabular}{ll}
\hline B The U(5) Formalism & 38
\end{tabular}

\section{Introduction and Summary}

Berkovits [1] recently proposed a new approach to covariantly quantise the superstring. This formalism has some clear advantageous features as compared to other, more traditional, approaches. For instance, one virtue is that it maintains the super-Poincaré symmetry of the superstring manifest, while avoiding problems associated with the quantisation of the Green-Schwarz (GS) superstring (essentially, the new formalism constructs the correct ghost sector for the classically covariant GS superstring). It also circumvents some of the more problematic aspects of the Ramond-Neveu-Schwarz (RNS) formalism, in particular the complications arising from the presence of spin fields in the vertex operators for the Ramond-Ramond (RR) fields and the spacetime fermions (as all important world-sheet fields in the new formalism have integer conformal weight, never producing branch cuts on the plane). The ghost degrees of freedom in Berkovits' approach involve certain constrained spinors, the so-called pure spinors. For this reason, the new formalism is often referred to as the "pure spinor superstring". Although a fair amount of research has been carried out since the appearance of [1], there are still many other 
aspects of the theory which one would like to understand better, such as its origin from sigma model gauge-fixing (see, e.g., [2, 3, 4]). Given the promise of the pure spinor formalism to by-pass the main difficulties in both GS and RNS formalisms, thus opening the way to previously unexplored aspects of superstring theory, it becomes very important to fully develop the pure spinor superstring in all its aspects. In this paper we shall take the first steps towards developing the boundary state operator formalism [5, 6, 7] (see, e.g., [8, 9] for reviews) in the pure spinor superstring (see [10] for earlier work in this direction).

The contents of this paper are as follows. In the next section we begin with a review of the pure spinor formalism and fix both our conventions and notation. We also show how the refined tree level zero mode saturation rule proposed recently in [11] can be obtained, starting from the well-known RNS expression. Then, in section 3. we present our proposal for the pure spinor boundary state in a flat spacetime background, discussing at length the most complicated part of this boundary state - its zero mode sector. In section 4 we propose a rule for calculating (tree level) scattering amplitudes in the presence of a D-brane and check that some results, which had been previously obtained using RNS methods, are correctly reproduced in the present framework. This serves as a consistency check on our proposal for the D-brane boundary state. We also discuss the coupling of the boundary state to the world-volume gauge field living on the D-brane. In particular, we check via scattering amplitude calculations that the Dirac-Born-Infeld (DBI) and Wess-Zumino (WZ) parts of the D-brane low-energy effective action are correctly reproduced. Again, this serves as a positive check on our proposal for the D-brane boundary state. Finally, in section 5 we summarise our results and discuss some possible directions for future research. In the appendices some more technical aspects are collected.

\section{Pure Spinors and the Covariant Superstring}

The covariant quantisation of the superstring using pure spinor ghosts was initiated by Berkovits in 11. For an introductory review we refer the reader to [12. Throughout this work we shall concentrate on the pure spinor version of the superstring in a flat spacetime background. Let us note that in this paper we will sometimes find it useful to compare expressions obtained for the pure spinor superstring to analogous ones in the RNS superstring, partly because this provides additional motivation for some of our expressions and partly because most readers are more familiar with the standard RNS formalism. Even though our comparisons with RNS have various levels of detail, they are mostly at the heuristic level (possibly with the exception of the discussion in section 2.2) and should thus not be thought of as rigourous derivations. It is probably possible to make some of our statements precise, by carefully implementing the map between RNS and pure spinor descriptions of the superstring given in [13. However, we believe that it is more important to develop and understand the pure spinor superstring formalism at the covariant level, and that our ultimate goal is to obtain a complete understanding of this theory, independently of its RNS counterpart. Thus, we shall leave our RNS derivations to be 
regarded mostly as motivations for the results we obtain. Another point we would like to stress is that in this paper we use bosonisation at certain intermediate stages of our considerations. However, in the final pure spinor expressions there is no need to bosonise the variables, a fact which has been previously argued to be one of the advantageous features of the pure spinor superstring formalism.

\subsection{Type II Covariant Superstrings in a Flat Background}

We begin by reviewing the pure spinor version of the superstring in a flat background, initiated in [1, and later developed in a long series of papers, e.g., [14, 15, 13, 16, 17, 18. We have attempted to make the presentation reasonably self-contained; more details can be found in the references.

In the pure spinor version of the type II superstring, the world-sheet fields are $\left(x^{m}, \theta^{\alpha}, \widetilde{\theta}^{\widetilde{\alpha}}\right)$ - the world-sheet analogues of the $\mathcal{N}=2, d=10$ superspace variablestogether with [19] $\left(p_{\alpha}, \widetilde{p}_{\widetilde{\alpha}}\right)$ where $p_{\alpha}$ is the conjugate momentum to $\theta^{\alpha}$ and $\widetilde{p}_{\widetilde{\alpha}}$ is the conjugate momentum to $\widetilde{\theta}^{\widetilde{\alpha}}$. Above $m=0, \ldots, 9$ and $\alpha, \widetilde{\alpha}=1, \ldots, 16$. In the type IIA superstring, where the target space has $(1,1)$ supersymmetry, $\alpha$ and $\widetilde{\alpha}$ denote $\mathrm{SO}(1,9)$ Majorana-Weyl spinors of opposite chirality, while in the type IIB superstring, where the target space has $(2,0)$ supersymmetry, $\alpha$ and $\widetilde{\alpha}$ denote $\mathrm{SO}(1,9)$ Majorana-Weyl spinors of the same chirality (in the case of the type IIB theory we shall later drop the ${ }^{\sim}$ on the $\widetilde{\alpha}$ indices to simplify the notation). In the pure spinor formalism, the world-sheet ghost fields are $\lambda^{\alpha}$ and $\widetilde{\lambda}^{\widetilde{\alpha}}$, which are complex bosonic Weyl spinors constrained to satisfy the pure spinor conditions [1]

$$
\lambda \gamma^{m} \lambda=0, \quad \tilde{\lambda} \gamma^{m} \widetilde{\lambda}=0 .
$$

The pure spinor conditions (2.1) reduce the number of independent complex components of both $\lambda^{\alpha}$ and $\widetilde{\lambda}^{\widetilde{\alpha}}$ from sixteen to eleven. It is important to notice that even though $\lambda^{\alpha}$ and $\widetilde{\lambda}^{\widetilde{\alpha}}$ are complex, they enter holomorphically in the theory (i.e., their complex conjugates, $\bar{\lambda}^{\alpha}$ and $\overline{\widetilde{\lambda}}^{\widetilde{\alpha}}$, never appear in the world-sheet action). This means that, e.g., in the determination of the central charge (see below) the counting of degrees of freedom is the same as if $\lambda^{\alpha}$ and $\widetilde{\lambda}^{\widetilde{\alpha}}$ were real. In (2.1), the gamma matrices $\gamma^{m}$ are the $16 \times 16$ off-diagonal blocks ("Pauli matrices") in the Weyl representation of the $32 \times 32$ ten-dimensional gamma matrices $\Gamma^{m}$. These two matrices are symmetric $\gamma_{\alpha \beta}^{m}=\gamma_{\beta \alpha}^{m}, \gamma^{m \alpha \beta}=\gamma^{m \beta \alpha}$, and satisfy

$$
\gamma_{\alpha \beta}^{m} \gamma^{n \beta \sigma}+\gamma_{\alpha \beta}^{n} \gamma^{m \beta \sigma}=2 \eta^{m n} \delta_{\alpha}^{\sigma}
$$

so that $\left\{\Gamma^{m}, \Gamma^{n}\right\}=2 \eta^{m n} \mathbb{1}_{32}$.

In a flat spacetime background, $\theta^{\alpha}, p_{\alpha}$ and $\lambda^{\alpha}$ are left-moving (holomorphic), while $\widetilde{\theta}^{\widetilde{\alpha}}, \widetilde{p}_{\widetilde{\alpha}}$ and $\widetilde{\lambda}^{\widetilde{\alpha}}$ are right-moving ${ }^{1}$ (anti-holomorphic). In the following we mostly only write the expressions involving the left-movers. The formulæ for the

\footnotetext{
${ }^{1}$ In many papers ${ }^{\wedge}$ (hat) is used instead of $\sim$ (tilde) to denote the right-moving variables.
} 
right-moving fields will look essentially the same. The world-sheet action in a flat background is (in units where $\alpha^{\prime}=2$ ) [1]

$$
S=\frac{1}{2 \pi} \int d^{2} z\left(\frac{1}{2} \partial x^{m} \bar{\partial} x_{m}+p_{\alpha} \bar{\partial} \theta^{\alpha}\right)+S_{\lambda},
$$

where $S_{\lambda}$ is the action for the $\lambda^{\alpha}$ ghosts. The free fields $x^{m}, \theta^{\alpha}$ and $p_{\alpha}$ have the standard OPE's,

$$
x^{m}(y, \bar{y}) x^{n}(z, \bar{z}) \sim-\eta^{m n} \log |y-z|^{2}, \quad p_{\alpha}(y) \theta^{\beta}(z) \sim \frac{\delta_{\alpha}^{\beta}}{y-z} .
$$

Because of the pure spinor constraint (2.1) the conjugate momenta to $\lambda^{\alpha}, w_{\alpha}$, is only defined modulo the transformation $w_{\alpha} \rightarrow w_{\alpha}+\left(\gamma^{m} \lambda\right)_{\alpha} \Lambda_{m}$. Although one could write an action for the ghost fields involving $w_{\alpha} \bar{\partial} \lambda^{\alpha}$ this form would be slightly deceiving since the pure spinor constraint implies, for example, that the OPE between $w_{\alpha}$ and $\lambda^{\beta}$ is not the canonical one, $c f$. (B.4). Nevertheless, it is still possible to work with this form of the ghost action provided one carefully takes into account the invariance under the transformation of $w_{\alpha}$ mentioned above (see, e.g., [20] for a discussion). Another possible way around the problem of the non-covariance of $w_{\alpha}$ is to relax the pure spinor constraint. This approach has been pursued in a number of works (see, e.g., 21, 22, 23] and references therein) but will not be further discussed in this paper. Yet another way to write down an explicit form for the ghost action, $S_{\lambda}$, in terms of free fields is to Wick rotate and to temporarily break the manifest $\mathrm{SO}(10)$ Lorentz invariance to $\mathrm{U}(5) \simeq \mathrm{SU}(5) \times \mathrm{U}(1)$ [1. As explained in [13, $\mathrm{U}(5)$ is the maximal subgroup of $\mathrm{SO}(10)$ which leaves the pure spinor constraint invariant.

As we have reviewed in appendix $\mathbb{B}$, an explicit parameterisation of $\lambda^{\alpha}$ satisfying the constraint (2.1) is 13

$$
\lambda^{+}=e^{s}, \quad \lambda_{a b}=u_{a b}, \quad \lambda^{a}=\frac{1}{8} e^{-s} \epsilon^{a b c d e} u_{b c} u_{d e},
$$

where $a=1, \ldots, 5$, and $u_{a b}=-u_{b a}$ are ten complex variables (which together with their conjugate momenta, $v^{a b}$, parameterise the $\mathrm{SO}(10) / \mathrm{U}(5)$ coset). The parameterisation (2.5) is well defined as long as $\lambda^{+} \neq 0$.

Using these "U(5) variables", the ghost action can be explicitly written as $^{2}$

$$
S_{\lambda}=\frac{1}{2 \pi} \int d^{2} z\left(v^{a b} \bar{\partial} u_{a b}+\beta^{\prime} \bar{\partial} \gamma^{\prime}\right),
$$

where $\beta^{\prime}$ has conformal weight 1 (let us stress that this $\beta \gamma$-system is not the standard one appearing in the RNS formulation). It turns out that it is convenient to bosonise $\left(\beta^{\prime}, \gamma^{\prime}\right)$ according to $\beta^{\prime} \cong e^{-\phi^{\prime}+\chi^{\prime}} \partial \chi^{\prime}$ and $\gamma^{\prime} \cong e^{\phi^{\prime}-\chi^{\prime}}$ and define $s=\frac{1}{2}\left(\chi^{\prime}-\phi^{\prime}\right)$ and $t=\chi^{\prime}+\phi^{\prime}$. It then follows that $\gamma^{\prime}=e^{-2 s}$. As we shall later see in greater detail,

\footnotetext{
${ }^{2}$ Note that the integrand equals $-w_{\alpha} \bar{\partial} \lambda^{\alpha}$ as can be seen using (B.3) with a suitable choice of the parameter $a$, together with the definitions of $\beta^{\prime}$ and $\gamma^{\prime}$ given below.
} 
this $s$ is the same as the one appearing in (2.5). The free fields $s, u_{a b}$, and their conjugate momenta $t, v^{a b}$, satisfy the free field OPE's,

$$
t(y) s(z) \sim \log (y-z), \quad v^{a b}(y) u_{c d}(z) \sim-\frac{\delta_{c d}^{a b}}{y-z},
$$

where $\delta_{c d}^{a b}=\frac{1}{2}\left(\delta_{c}^{a} \delta_{d}^{b}-\delta_{d}^{a} \delta_{c}^{b}\right)$.

The holomorphic stress tensor in the pure spinor superstring is

$$
T=-\frac{1}{2} \partial x^{m} \partial x_{m}-p_{\alpha} \partial \theta^{\alpha}+T_{\lambda},
$$

where $T_{\lambda}$ is the stress tensor for the $\lambda^{\alpha}$ ghosts. In terms of the free $\mathrm{U}(5)$ variables, the ghost stress tensor can be written as (later we show that $\partial t \partial s+\partial^{2} s=-\beta^{\prime} \partial \gamma^{\prime}$ )

$$
T_{\lambda}=-v^{a b} \partial u_{a b}+\partial t \partial s+\partial^{2} s .
$$

Using this expression it can be readily checked that the ghost CFT has $c=22$. If one recalls that the $x^{m} \mathrm{CFT}$ has the standard $c=10$ central charge, while the $(p, \theta)$ CFT has central charge $c=-32$, the total central charge vanishes as required.

Even though the OPE between $w_{\alpha}$ and $\lambda^{\alpha}$ is not manifestly $\mathrm{SO}(10)$ covariant, one can nevertheless explicitly construct $\mathrm{SO}(10)$ Lorentz currents for the ghosts as [1]

$$
N^{m n}=\frac{1}{2} w \gamma^{m n} \lambda \text {. }
$$

As shown in 1, and discussed at greater length in appendix B, the OPE's involving $N_{m n}$ and $\lambda^{\alpha}$ have the manifestly $\mathrm{SO}(10)$ covariant form

$$
\begin{aligned}
N^{m n}(y) \lambda^{\alpha}(z) & \sim \frac{1}{2} \frac{1}{y-z}\left(\gamma^{m n}\right)_{\beta}^{\alpha} \lambda^{\beta}(z), \\
N^{p q}(y) N^{m n}(z) & \sim \frac{\eta^{p m} N^{q n}(z)-\eta^{q m} N^{p n}(z)-(m \leftrightarrow n)}{y-z}-3 \frac{\eta^{p n} \eta^{q m}-\eta^{p m} \eta^{q n}}{(y-z)^{2}} .
\end{aligned}
$$

From this expression we see that the ghost Lorentz currents $N^{m n}$ form a $\mathrm{SO}(10)$ current algebra with level $k=-3$. In comparison, the OPE's involving the $(p, \theta)$ Lorentz current, $M^{m n}=-\frac{1}{2} p \gamma^{m n} \theta$, take the form

$$
\begin{aligned}
M^{m n}(y) \theta^{\alpha}(z) & \sim \frac{1}{2} \frac{1}{y-z}\left(\gamma^{m n}\right)_{\beta}^{\alpha} \theta^{\beta}(z), \\
M^{p q}(y) M^{m n}(z) & \sim \frac{\eta^{p m} M^{q n}(z)-\eta^{q m} M^{p n}(z)-(m \leftrightarrow n)}{y-z}+4 \frac{\eta^{p n} \eta^{q m}-\eta^{p m} \eta^{q n}}{(y-z)^{2}} .
\end{aligned}
$$

Thus the $M^{m n}$ 's also form a $\mathrm{SO}(10)$ current algebra, this time at level $k=4$. The total Lorentz current $L_{m n}=-\frac{1}{2} p \gamma^{m n} \theta+N^{m n}$ satisfies the OPE

$$
L^{p q}(y) L^{m n}(z) \sim \frac{\eta^{p m} L^{q n}(z)-\eta^{q m} L^{p n}(z)-(m \leftrightarrow n)}{y-z}+\frac{\eta^{p n} \eta^{q m}-\eta^{p m} \eta^{q n}}{(y-z)^{2}}
$$


and thus forms a current algebra with level $k=1$ as expected from comparison with the RNS formalism [1, where the $L^{m n}=-\Psi^{m} \Psi^{n}$ satisfy (2.13).

As should be clear from the above discussion, the OPE's of the fields $\lambda^{\alpha}$ and the currents $N^{m n}$ are manifestly covariant (even though they were computed starting from the non-covariant free ghost action). Moreover, although one can in principle write down pure spinor vertex operators in terms of the free variables, it turns out that the requirement of super-Poincaré covariance implies that the free variables $\left\{s, t, u_{a b}, v^{a b}\right\}$ can only appear in the Lorentz covariant combinations of $\left\{\lambda^{\alpha}, N^{m n}, \partial h\right\}$, where $h$ is a Lorentz scalar defined through

$$
\partial h=\frac{1}{2} w_{\alpha} \lambda^{\alpha}
$$

As was the case for $N^{m n}$, even though $\partial h$ contains the "non-covariant" quantity $w_{\alpha}$, the OPE's involving $h, N^{m n}$ and $\lambda^{\alpha}$ are manifestly Lorentz covariant. Observe that both $N^{m n}$ and $\partial h$ are invariant under the transformation $w_{\alpha} \rightarrow w_{\alpha}+\left(\gamma^{m} \lambda\right)_{\alpha} \Lambda_{m}$, because of the pure spinor constraint on $\lambda^{\alpha}$. The Lorentz scalar $\partial h$ has no singularities with the Lorentz currents $N^{m n}$ and satisfies the OPE's

$$
h(y) h(z) \sim-\log (y-z), \quad \partial h(y) \lambda^{\alpha}(z) \sim \frac{1}{2} \frac{1}{y-z} \lambda^{\alpha}(z) .
$$

It is possible to show that the operator $J=2 \oint \partial h$ is the ghost number charge, implying that $\lambda^{\alpha}$ has ghost number one as it should have.

Furthermore, using the covariant fields $N^{m n}$ and $\partial h$, the ghost stress tensor can be written in a manifestly Lorentz invariant way as 13

$$
T_{N, \partial h}=-\frac{1}{20} N_{m n} N^{m n}-\frac{1}{2}(\partial h)^{2}+2 \partial^{2} h .
$$

By using various normal ordering rearrangements, one can check that the stress tensor (2.16) indeed reduces to the stress tensor (2.9) written in terms of the $\mathrm{U}(5)$ variables, $s, t, u_{a b}$ and $v^{a b}$ (see appendix $\mathrm{B}$ for further details). Let us analyse the ghost stress tensor (2.16) in more detail. The first piece involves the ghost Lorentz currents, $N^{m n}$, and is a Sugawara construction for a $\mathrm{SO}(10)$ WZNW model at level $k=-3$. Indeed, recalling that the dual Coxeter number of $\mathrm{SO}(10)$ is $g=8$, we find $^{3} 2(g+k)=10$. The second piece refers to a Coulomb gas, with a background charge of $Q=4$. Using standard formulæ one finds that the central charge of the ghost Lorentz currents is

$$
c=\frac{k \operatorname{dim} \mathrm{SO}(10)}{k+g}=\frac{(-3)(45)}{-3+8}=-27,
$$

while the central charge of the Coulomb gas field is $c=1+3 Q^{2}=49$, so that $-27+$ $49=22$ as expected. The expression for the stress tensor (2.16) also allows one to write explicitly covariant expressions for the ghost action, albeit in the complicated form as a sum of a WZNW and a Coulomb gas action.

\footnotetext{
${ }^{3}$ Due to our normalisation of the $N N$ OPE in (2.11) the prefactor in front of $N_{m n} N^{m n}$ in (2.16) is unconventional. To obtain the usual $+\frac{1}{10}$ one would have to rescale the currents $N^{m n}$.
} 
To obtain a better understanding of the form of the pure spinor stress tensor we note that it is also possible to rewrite the $(p, \theta)$ stress tensor in the form

$$
T_{M, \partial g}=-\frac{1}{48} M_{m n} M^{m n}+\frac{1}{2}(\partial g)^{2}-2 \partial^{2} g
$$

where, as above, $M^{m n}=-\frac{1}{2} p \gamma^{m n} \theta$ and satisfies the OPE's (2.12), while the Lorentz scalar $\partial g=\frac{1}{4} p_{\alpha} \theta^{\alpha}$ has no singularities with $M^{m n}$ and satisfies the OPE's

$$
g(y) g(z) \sim \log (y-z), \quad \partial g(y) \theta^{\alpha}(z) \sim-\frac{1}{4} \frac{1}{y-z} \theta^{\alpha}(z) .
$$

In the $(p, \theta)$ stress tensor, the spacetime fermion Lorentz currents, $M^{m n}$, appear as a Sugawara construction of a $\mathrm{SO}(10)$ WZNW model at level $k=4$, and the Coulomb gas piece has background charge $Q=4$. Further observe that the operator $K=4 \oint \partial g$ is the spacetime fermion number charge, implying that $\theta^{\alpha}$ has fermion number -1 . In the form (2.18) the central charge is calculated as $4 \cdot 45 / 12+(1-$ $\left.3 \cdot 4^{2}\right)=15-47=-32$. It is a straightforward but tedious exercise to show that after using various normal ordering rearrangements the stress tensor (2.18) indeed reduces to $T_{p \theta}=-p_{\alpha} \partial \theta^{\alpha}$.

We have thus learned that the full stress tensor (excluding the $\partial x$ part) can be written as a sum of two WZNW and two Coulomb gas pieces. In 24, 23] it has also been noted that the pure spinor superstring (in the extended formulation where the pure spinor constraint is relaxed) can be formulated in terms of WZNW models.

The above discussion can be summarised in the following table, containing the conformal weights and ghost number assignments of the world-sheet fields:

\begin{tabular}{|c|c|c|}
\hline Field & Conformal Dimension & Ghost Number \\
\hline$\partial x^{m}, p_{\alpha}$ & 1 & 0 \\
\hline$\theta^{\alpha}$ & 0 & 0 \\
\hline$\lambda^{\alpha}$ & 0 & 1 \\
\hline$M^{m n}, N^{m n}$ & 1 & 0 \\
\hline$\partial g, \partial h$ & $1^{*}$ & 0 \\
\hline
\end{tabular}

Table 1: Conformal dimensions and ghost numbers of the various fields. The ${ }^{*}$ indicates that $\partial g$ and $\partial h$ do not have honest conformal dimensions because of their background charges.

These properties can be derived using both the free field form of the above stress tensors or the WNZW/Coulomb gas one, but depending on what one wants to calculate it is usually easier to use one of the two (equivalent) versions.

The physical states of the superstring in the pure spinor formalism are obtained from vertex operators in the cohomology of the left- and right-moving BRST operators [1]

$$
Q=\oint \lambda^{\alpha} d_{\alpha}, \quad \widetilde{Q}=\oint \widetilde{\lambda}^{\widetilde{\alpha}} \widetilde{d}_{\widetilde{\alpha}},
$$

where (the definition of $\widetilde{d}_{\widetilde{\alpha}}$ is completely analogous)

$$
d_{\alpha}=p_{\alpha}-\frac{1}{2}\left(\gamma^{m} \theta\right)_{\alpha} \partial x_{m}-\frac{1}{8}\left(\gamma^{m} \theta\right)_{\alpha}\left(\theta \gamma_{m} \partial \theta\right)
$$


The world-sheet field $d_{\alpha}$ satisfies the OPE's ${ }^{4}$

$$
\begin{aligned}
& d_{\alpha}(y) d_{\beta}(z) \sim-\frac{1}{y-z} \gamma_{\alpha \beta}^{m} \Pi_{m}(z), \quad d_{\alpha}(y) \Pi^{m}(z) \sim \frac{1}{y-z}\left(\gamma^{m} \partial \theta\right)_{\alpha}(z), \\
& d_{\alpha}(y) \partial \theta^{\beta}(z) \sim \frac{1}{(y-z)^{2}} \delta_{\alpha}^{\beta},
\end{aligned}
$$

where

$$
\Pi^{m}=\partial x^{m}+\frac{1}{2} \theta \gamma^{m} \partial \theta .
$$

Using the above OPE's it is easy to check that the BRST operator is nilpotent (due to the pure spinor condition (2.1) ), so that $Q^{2}=0=\widetilde{Q}^{2}$.

The spacetime supersymmetry generator is

$$
q_{\alpha}=\oint\left(p_{\alpha}+\frac{1}{2}\left(\gamma^{m} \theta\right)_{\alpha} \partial x_{m}+\frac{1}{24}\left(\gamma^{m} \theta\right)_{\alpha}\left(\theta \gamma_{m} \partial \theta\right)\right)
$$

and satisfies the anticommutation relation

$$
\left\{q_{\alpha}, q_{\beta}\right\}=\gamma_{\alpha \beta}^{m} \oint \partial x_{m} .
$$

It is straightforward to check that the BRST operator as well as the world-sheet fields $d_{\alpha}, \Pi^{m}$ are supersymmetric, i.e., they (anti)commute with $q_{\alpha}$.

For an arbitrary superfield $\Phi(x, \theta)$, one has that $\left[\oint d_{\alpha}, \Phi(x, \theta)\right\}=D_{\alpha} \Phi(x, \theta)$ where ${ }^{5}$

$$
D_{\alpha}=\frac{\partial}{\partial \theta^{\alpha}}+\frac{1}{2}\left(\gamma^{m} \theta\right)_{\alpha} \frac{\partial}{\partial x^{m}}
$$

Thus, the world-sheet field $d_{\alpha}$ corresponds to the spacetime supersymmetric covariant derivative. Similarly, one has $\left[q_{\alpha} \Phi(x, \theta)\right\}=\mathcal{Q}_{\alpha} \Phi(x, \theta)$, where

$$
\mathcal{Q}_{\alpha}=\frac{\partial}{\partial \theta^{\alpha}}-\frac{1}{2}\left(\gamma^{m} \theta\right)_{\alpha} \frac{\partial}{\partial x^{m}}
$$

Vertex operators in flat space were constructed in 11, 14, 18, 25, 26. The unintegrated vertex operator for the massless open superstring state is $U=\lambda^{\alpha} A_{\alpha}(x, \theta)$, where $A_{\alpha}(x, \theta)$ is a superfield. Since $\left\{Q, A_{\beta}(x, \theta)\right\}=\lambda^{\alpha} D_{\alpha} A_{\beta}(x, \theta)$, the physical requirement $\{Q, U\}=0$ implies the ten-dimensional (superspace) Yang-Mills equations of motion (using that for pure spinors $\lambda^{\alpha} \lambda^{\beta}=\frac{1}{1920} \gamma_{m_{1} \cdots m_{5}}^{\alpha \beta} \lambda \gamma^{m_{1} \cdots m_{5}} \lambda$ )

$$
\gamma_{m_{1} \cdots m_{5}}^{\alpha \beta} D_{\alpha} A_{\beta}=0
$$

Hence $A_{\alpha}$ is the spinor superfield potential for super Yang-Mills theory (see appendix $\mathrm{A}$ for more details). Moreover, the BRST invariance $\delta U=[Q, \Omega]$ implies the

\footnotetext{
${ }^{4}$ In order to verify the first of these OPE's, it is crucial to use the gamma matrix identity $\left(\gamma^{m} \theta\right)_{\alpha}\left(\gamma_{m} \xi\right)_{\beta}=\frac{1}{2}\left[\left(\gamma^{m} \theta\right)_{\alpha}\left(\gamma_{m} \xi\right)_{\beta}-\left(\gamma^{m} \theta\right)_{\beta}\left(\gamma_{m} \xi\right)_{\alpha}\right]-\frac{1}{2} \gamma_{\alpha \beta}^{m}\left(\theta \gamma_{m} \xi\right)$
}

${ }^{5}$ We refer the reader to appendix $\mathrm{A}$ for our superspace conventions. 
Yang-Mills gauge transformations $\delta A_{\alpha}=D_{\alpha} \Omega$. Choosing an appropriate gauge and restricting to constant field strengths, one can write (see also appendix A)

$$
U=\frac{1}{2}\left(\lambda \gamma^{m} \theta\right) a_{m}(x)+\frac{1}{3}\left(\lambda \gamma^{m} \theta\right)\left(\theta \gamma_{m} \xi\right)-\frac{1}{32}\left(\lambda \gamma_{\sigma} \theta\right)\left(\theta \gamma^{\sigma m n} \theta\right) f_{m n}
$$

where $a_{m}(x)$ is the gluon with constant field strength $f_{m n}$, and $\xi^{\alpha}$ is the constant gluino. For non-constant fields there will be additional terms in (2.29).

Integrated vertex operators, $\oint V$, are defined through $[Q, V]=\partial U$, and are thus manifestly BRST invariant. For the massless states of the open superstring,

$$
V=\Pi^{m} A_{m}(x, \theta)+\partial \theta^{\alpha} A_{\alpha}(x, \theta)+d_{\alpha} W^{\alpha}(x, \theta)+\frac{1}{2} N^{m n} F_{m n}(x, \theta) .
$$

The definitions of the superfields $A_{m}, W^{\alpha}$ and $F_{m n}$ can be found in appendix A To lowest order in the component fields, and in the same gauge as before,

$$
V=a_{m}(x) \partial x^{m}+\frac{1}{2} f_{m n}\left(-\frac{1}{2} p \gamma^{m n} \theta+N^{m n}\right)+\xi^{\alpha} q_{\alpha}
$$

For the closed superstring the story is similar (see [26] for an extensive recent discussion). The massless unintegrated vertex operator is $U=\lambda^{\alpha} \widetilde{\lambda}^{\widetilde{\beta}} A_{\alpha \widetilde{\beta}}(x, \theta, \widetilde{\theta})$, where $A_{\alpha \widetilde{\beta}}$ is a $\mathcal{N}=2, d=10$ bispinor superfield. The physical state conditions are ${ }^{6}$ $[Q, U]=0=[\widetilde{Q}, U]$ implying the (linearised) supergravity equations of motion, and the gauge invariance is $\delta U=\{Q, \widetilde{\Omega}\}+\{\widetilde{Q}, \Omega\}$ with $\{\widetilde{Q}, \widetilde{\Omega}\}=0=\{Q, \Omega\}$. In order to construct the component forms of the massless closed string vertex operators, the simplest way to proceed is to use the fact that these operators can be understood as the left-right product of the open string vertex operators that we described above. The vertex operator,

$$
\lambda A \widetilde{\lambda}=\left(\lambda \gamma^{m} \theta\right) \zeta_{m n}(x)\left(\widetilde{\lambda} \gamma^{n} \widetilde{\theta}\right),
$$

with the gauge choice $k^{m} \zeta_{m n}=0, k^{2} \zeta_{m n}=0$, describes a graviton when $\zeta_{m n}=$ $h_{m n}=h_{n m}$ with $\eta^{m n} h_{m n}=0$. It describes a $B$-field when $\zeta_{m n}=B_{m n}=-B_{n m}$ and it describes the dilaton when $\zeta_{m n}(x)=\zeta(x) \epsilon_{m n}$ and

$$
\epsilon_{m n}=\frac{1}{\sqrt{d-2}}\left(\eta_{m n}-k_{m} \ell_{n}-k_{n} \ell_{m}\right), \quad k \cdot \ell=1, \quad \ell \cdot \ell=0 .
$$

In the above equations, $\zeta_{m n}(x)=\zeta_{m n}(k) e^{i k \cdot x}$ and $k^{m}$ is the spacetime momentum. In order to describe spacetime fermions, one should use either the vertex operator

$$
\lambda A \widetilde{\lambda}=\left(\lambda \gamma^{m} \theta\right)\left(\Upsilon_{n}(x) \gamma_{m} \theta\right)\left(\widetilde{\theta} \gamma^{n} \widetilde{\lambda}\right),
$$

or the vertex operator

$$
\lambda A \widetilde{\lambda}=\left(\lambda \gamma^{m} \theta\right)\left(\theta \gamma_{n} \widetilde{\Upsilon}_{m}(x)\right)\left(\widetilde{\theta} \gamma^{n} \widetilde{\lambda}\right),
$$

\footnotetext{
${ }^{6}$ In this paper we shall ignore the subtleties associated with zero momentum states.
} 
where the spacetime fields $\Upsilon_{m}^{\alpha}$ and $\widetilde{\Upsilon}_{m}^{\widetilde{\alpha}}$ describe the gravitini and the dilatini. For $\mathrm{RR}$ fields one conventionally uses the vertex operator

$$
\lambda A \widetilde{\lambda}=\left(\lambda \gamma^{m} \theta\right)\left(\theta \gamma_{m} F(x) \gamma_{n} \widetilde{\theta}\right)\left(\widetilde{\theta} \gamma^{n} \widetilde{\lambda}\right),
$$

where $F$ is the RR field strength,

$$
F=\sum_{n} \frac{1}{n !} \gamma^{m_{1}} \cdots \gamma^{m_{n}} F_{m_{1} \cdots m_{n}}(x) .
$$

Here $n$ is even in type IIA and odd in type IIB. The vertex operator associated with a constant RR gauge field $C$ has also been studied [25] (see also [26]), and is

$$
\lambda A \widetilde{\lambda}=\left(\lambda \gamma^{m} \theta\right)\left(\theta \gamma_{m} C \widetilde{\lambda}\right)-\left(\lambda C \gamma_{m} \widetilde{\theta}\right)\left(\widetilde{\theta} \gamma^{m} \widetilde{\lambda}\right)
$$

where

$$
C=\sum_{n} \frac{1}{n !} \gamma^{m_{1}} \cdots \gamma^{m_{n}} C_{m_{1} \cdots m_{n}}
$$

In this expression $n$ is even in type IIB and odd in type IIA. For later reference we note that in type IIB the vertex operator can also be written as

$$
\lambda A \widetilde{\lambda}=-\left(\widetilde{\lambda} \widetilde{C} \gamma_{m} \theta\right)\left(\theta \gamma^{m} \lambda\right)-\left(\lambda C \gamma_{m} \widetilde{\theta}\right)\left(\widetilde{\theta} \gamma^{m} \widetilde{\lambda}\right)
$$

where $\widetilde{C}_{\alpha}{ }^{\beta}=C^{\beta}{ }_{\alpha}$, or explicitly,

$$
\widetilde{C}_{\alpha}^{\beta}=\sum_{n \text { even }} \frac{(-1)^{\frac{n}{2}}}{n !}\left(\gamma^{m_{1} \cdots m_{n}}\right)_{\alpha}{ }^{\beta} C_{m_{1} \cdots m_{n}} .
$$

The integrated vertex operators can also be constructed in the same way as for the open superstring. For instance, the integrated vertex operator (in momentum space) corresponding to the Neveu-Schwarz-Neveu-Schwarz (NSNS) fields is given by

$$
V \propto \zeta_{m n}\left(\partial X^{m}+i k_{r} L^{r n}\right)\left(\bar{\partial} x^{n}+i k_{s} \widetilde{L}^{s m}\right) e^{i k \cdot X}+\cdots
$$

As before, $\zeta_{m n}$ equals $h_{m n}, B_{m n}$ or $\epsilon_{m n} \zeta$, and the dots refer to terms with additional $\theta$ 's and additional powers of $k$. Above we only discussed the massless modes of the string; vertex operators for the first massive level have been studied in [18.

To compute tree amplitudes one also needs to deal with the zero modes of $\theta^{\alpha}$ and $\lambda^{\alpha}$. It was argued in 13 that the pure spinor analogue of the RNS operator

$$
c \partial c \partial^{2} c e^{-2 \phi}
$$

which saturates the zero modes in tree amplitudes [27] is

$$
\left(\lambda \gamma_{m} \theta\right)\left(\lambda \gamma_{n} \theta\right)\left(\lambda \gamma_{p} \theta\right)\left(\theta \gamma^{m n p} \theta\right)
$$


This is the unique element of ghost number three in the cohomology of the BRST operator (2.20) [1. Tree amplitudes are then obtained via $n$-point correlation functions with three unintegrated vertex operators and $n-3$ integrated vertex operators, such that the zero modes of $\theta^{\alpha}$ and $\lambda^{\alpha}$ are saturated via the correlator [1]

$$
\left\langle\left(\lambda \gamma^{m} \theta\right)\left(\lambda \gamma^{n} \theta\right)\left(\lambda \gamma^{p} \theta\right)\left(\theta \gamma_{m n p} \theta\right)\right\rangle=\text { const. }
$$

It has been checked that this prescription leads to results which are in complete agreement with the ones obtained from RNS 14. Nevertheless, there remain some puzzling aspects about the saturation rule (2.45). Some of those puzzles were resolved in the recent paper [11] where a refinement of the saturation rule was proposed. In the following, we shall show that this refinement can be obtained naturally by starting from the RNS saturation operator (2.43).

\subsection{Berkovits' Tree Level Zero Mode Saturation Rule from RNS}

There is a seeming discrepancy between the (tree level) saturation rule (2.45) for the zero modes in the pure spinor superstring, which we shall schematically write as $\left\langle\lambda^{3} \theta^{5}\right\rangle \neq 0$, and the one obtained by analysing (using the standard methods in [27]) the background charges of $\partial h$ and $\partial g$ in (2.16), (2.18). In fact, from (2.15) and (2.19), it follows that $\lambda^{\alpha}$ has $\partial h$ charge $\frac{1}{2}$ while $\theta^{\alpha}$ has $\partial g$ charge $-\frac{1}{4}$. Together with the fact that both Coulomb gas fields have background charge 4 , this naturally leads to the schematic saturation rule $\left\langle\lambda^{-8} \theta^{16}\right\rangle \neq 0$ cancelling both background charges, but in contradiction with the previous expression. Recently Berkovits [1] proposed a refined version of the original saturation rule which resolves this mismatch: to relate the two saturation rules one further needs to insert eleven operators $Y^{I}$ (defined in [11]) into the original definition, each of which carries $(\lambda, \theta)$ charge $(-1,+1)$. Importantly, the properties of these operators are such that they do not affect earlier considerations in the literature based on the original rule (2.45) and thus, for most purposes, they can be ignored. We should also point out that in earlier work by Chesterman [28] equivalent, but less explicit, results were also obtained. In his work the existence of the different saturation rules was explained at the level of cohomology as arising from the isomorphism of certain cohomologies. In this language the extra $Y^{I}$ insertions can be viewed as the map implementing the isomorphism.

In this subsection we show how the modified saturation rule given in [1] arises from the saturation rule in the RNS superstring, using the map relating the two formulations [13]. Throughout this section we shall not keep track of numerical factors as they are not important for our conclusions. We will also only write the expressions for the left-moving sector explicitly; the right-moving sector is completely analogous. To begin with, recall the bosonisation formulæ of the RNS ghost variables $(\beta, \gamma, b, c)$ :

$$
\beta=\partial \xi e^{-\phi}, \quad \gamma=\eta e^{\phi}, \quad \xi=e^{\chi}, \quad \eta=e^{-\chi}, \quad c=e^{\sigma}, \quad b=e^{-\sigma},
$$


from which it follows that (see, e.g., [29])

$$
: b c:=-\partial \sigma, \quad: \xi \eta:=\partial \chi, \quad \delta(\gamma)=e^{-\phi}, \quad \delta(\beta)=e^{\phi} .
$$

The bosonisation of the RNS world-sheet fermion $\Psi^{m}$ is (here $a=1, \ldots, 5$ )

$$
\Psi^{a} \pm i \Psi^{a+5}=e^{\mp \tau^{a}} .
$$

Later we will use the notation $\psi^{a}=e^{-\tau^{a}}$ and $\psi_{a}=e^{\tau^{a}}$ As usual [27] one constructs the spin fields from $e^{ \pm \tau^{1} \pm \tau^{2} \pm \tau^{3} \pm \tau^{4} \pm \tau^{5}}$ where the 32 different possibilities span a 32 dimensional Dirac spinor, which decomposes into a Weyl spinor $S^{\alpha}$, and an antiWeyl spinor $S_{\alpha}$. These in turn decompose into $\left(S^{+}, S^{a}, S_{a b}\right)$ and $\left(S_{+}, S_{a}, S^{a b}\right)$ under the U(5) subgroup (see appendix $\mathbb{B}$ for further details). Here we will only need

$$
S^{a}=e^{-\tau^{a}+\sum_{b} \tau^{b} / 2}, \quad S_{a}=e^{\tau^{a}-\sum_{b} \tau^{b} / 2}, \quad S^{+}=e^{-\sum_{a} \tau^{a} / 2}, \quad S_{+}=e^{\sum_{a} \tau^{a} / 2} .
$$

In the RNS theory, the well known saturation rules for the zero modes are (the first expression is just the bosonised version of (2.43) $)$

$$
\begin{aligned}
\left\langle e^{3 \sigma-2 \phi}\right\rangle \neq 0 & \text { (small Hilbert space) }, \\
\left\langle e^{3 \sigma-2 \phi+\chi}\right\rangle \neq 0 & \text { (large Hilbert space) } .
\end{aligned}
$$

To relate these expressions to the pure spinor result we first make the change of variables from RNS to the $\mathrm{U}(5)$ variables introduced in [30, 13] by Berkovits. In terms of these variables, a $\mathrm{U}(5)$ subgroup of the (Wick-rotated) $\mathrm{SO}(10)$ superPoincaré symmetry is manifest [30. The U(5) variables comprise ${ }^{7} 12$ Grassmannodd variables

$$
\theta^{a}=e^{\phi / 2} S^{a}, \quad \theta^{+}=c \xi e^{-3 \phi / 2} S^{+}, \quad p_{a}=e^{-\phi / 2} S_{a}, \quad p_{+}=b \eta e^{3 \phi / 2} S_{+},
$$

as well as the two Grassmann-even ones

$$
s=\sigma-\frac{3}{2} \phi-\frac{1}{2} \sum_{a=1}^{5} \tau^{a}, \quad t=-\chi+\frac{3}{2} \phi+\frac{1}{2} \sum_{a=1}^{5} \tau^{a} .
$$

In the $(s, t)$ sector, the OPE is as (2.7) and the energy-momentum tensor is

$$
T=\partial s \partial t+\partial^{2} s
$$

By redefining these variables according to

$$
s=\frac{1}{2}\left(\chi^{\prime}-\phi^{\prime}\right), \quad t=\chi^{\prime}+\phi^{\prime},
$$

\footnotetext{
${ }^{7}$ As shown in 13 these $\mathrm{U}(5)$ variables are the same as (a subset of) the $\mathrm{U}(5)$ variables discussed in section 2.1. As in [13] the missing quartet $\left(p^{a b}, \theta_{a b}, v^{a b}, u_{a b}\right)$ will be added later.
} 
one finds

$$
T=\frac{1}{2} \partial \chi^{\prime} \partial \chi^{\prime}+\frac{1}{2} \partial^{2} \chi^{\prime}-\frac{1}{2} \partial \phi^{\prime} \partial \phi^{\prime}-\frac{1}{2} \partial^{2} \phi^{\prime},
$$

which one recognises (see, e.g., [29]) as a bosonised $\beta^{\prime} \gamma^{\prime}$-system with weight $\lambda^{\prime}=1$ $\left(\lambda=\frac{3}{2}\right)$ and therefore $T=-\beta^{\prime} \partial \gamma^{\prime}$. Note that this is not the same as the usual RNS $\beta \gamma$-system. In particular, the conformal weight is different. The complete energymomentum tensor also includes $T=-p_{a} \partial \theta^{a}-p_{+} \partial \theta^{+}$, i.e., six $b c$-type systems, all of weight one. In the large Hilbert space (with respect to the $\beta^{\prime} \gamma^{\prime}$-system) the saturation rule becomes (using standard methods [27])

$$
\left\langle e^{\chi^{\prime}-\phi^{\prime}} \epsilon_{a b c d e} \theta^{a} \theta^{b} \theta^{c} \theta^{d} \theta^{e} \theta^{+}\right\rangle \neq 0 \quad \leftrightarrow \quad\left\langle\xi^{\prime} \delta\left(\gamma^{\prime}\right) \epsilon_{a b c d e} \theta^{a} \theta^{b} \theta^{c} \theta^{d} \theta^{e} \theta^{+}\right\rangle \neq 0
$$

Translating to the $(s, t)$ variables this becomes

$$
\left\langle e^{2 s} \epsilon_{a b c d e} \theta^{a} \theta^{b} \theta^{c} \theta^{d} \theta^{e} \theta^{+}\right\rangle \neq 0,
$$

which agrees with what one obtains by translating the RNS result (2.50) in the large Hilbert space (with respect to the $\beta \gamma$-system), using the change of variables in (2.51) and (2.52). This was to be expected since as long as one includes all zero modes (i.e., one works in the large Hilbert space) the saturation rule should be the same no matter which variables are used. On the other hand, in the small Hilbert spaces the saturation rules will not agree in general since they are defined with respect to different $\beta \gamma$-systems. In the small $\mathrm{U}(5)$ Hilbert space (with respect to the $\beta^{\prime} \gamma^{\prime}$-system) the saturation rule is

$$
\left\langle e^{-\phi^{\prime}} \epsilon_{a b c d e} \theta^{a} \theta^{b} \theta^{c} \theta^{d} \theta^{e} \theta^{+}\right\rangle \neq 0 \quad \leftrightarrow \quad\left\langle\delta\left(\gamma^{\prime}\right) \epsilon_{a b c d e} \theta^{a} \theta^{b} \theta^{c} \theta^{d} \theta^{e} \theta^{+}\right\rangle \neq 0
$$

which does not agree with what one obtains by translating the RNS result (2.50) in the small RNS Hilbert space (with respect to the $\beta \gamma$-system), using the corresponding change of variables.

In the map between RNS and pure spinor formulations [13, the first step is to move to the large RNS Hilbert space. One would thus expect that after the change of variables it is the large $\mathrm{U}(5)$ Hilbert space which is relevant. However, one is also free to move to the small U(5) Hilbert space, and we will argue below that this is required in order to obtain the $\mathrm{SO}(10)$ covariant result.

To proceed, we add [13] the "topological" quartet $\left(p^{a b}, \theta_{a b}, v^{a b}, u_{a b}\right)$ (cf. section 2.1). This is a sum of ten $b c$ - and ten $\beta \gamma$-type systems all with weight one. The additional operator insertion needed to saturate the zero modes is therefore:

$$
\prod_{a b=1}^{10} \theta_{a b} \delta\left(u_{a b}\right)
$$


Note that this operator is in the small Hilbert spaces (with respect to the $\left(v^{a b}, u_{a b}\right)$ systems). Combining the above results it follows that the saturation rule can be written as

$$
\left\langle\epsilon_{a b c d e} \theta^{a} \theta^{b} \theta^{c} \theta^{d} \theta^{e}\left[\theta^{+} \prod_{a b=1}^{10} \theta_{a b}\right]\left[\delta\left(\gamma^{\prime}\right) \prod_{a b=1}^{10} \delta\left(u_{a b}\right)\right]\right\rangle \neq 0 .
$$

From (2.54) and the discussion below (2.6) we have $\gamma^{\prime}=e^{-2 s}$, whereas from (2.5) we find $\lambda^{+}=e^{s}=\gamma^{\prime-1 / 2}$. Using the relation $\delta(x)=f^{\prime}(x) \delta(f(x))$ we find ${ }^{8} \delta\left(\gamma^{\prime}\right) \propto$ $\left(\lambda^{+}\right)^{3} \delta\left(\lambda^{+}\right)$. Using this result together with (2.5) we finally find

$$
\left\langle\left(\lambda^{+}\right)^{3} \epsilon_{a b c d e} \theta^{a} \theta^{b} \theta^{c} \theta^{d} \theta^{e}\left[\theta^{+} \prod_{a b=1}^{10} \theta_{a b}\right]\left[\delta\left(\lambda^{+}\right) \prod_{a b=1}^{10} \delta\left(\lambda_{a b}\right)\right]\right\rangle \neq 0,
$$

which is equivalent to

$$
\left\langle\left(\lambda \gamma^{m} \theta\right)\left(\lambda \gamma^{n} \theta\right)\left(\lambda \gamma^{p} \theta\right)\left(\theta \gamma_{m n p} \theta\right)\left[\theta^{+} \prod_{a b=1}^{10} \theta_{a b}\right]\left[\delta\left(\lambda^{+}\right) \prod_{a b=1}^{10} \delta\left(\lambda_{a b}\right)\right]\right\rangle \neq 0
$$

because the extra terms are set to zero by the delta functions (recall that for Grassmann-odd variables, $\delta(\theta)=\theta$ ). One can write (2.62) as

$$
\left.\left\langle\left(\lambda \gamma^{m} \theta\right)\left(\lambda \gamma^{n} \theta\right)\left(\lambda \gamma^{p} \theta\right)\left(\theta \gamma_{m n p} \theta\right) \prod_{I=1}^{11} C_{\alpha}^{I} \theta^{\alpha} \delta\left(C_{\beta}^{I} \lambda^{\beta}\right)\right]\right\rangle \neq 0,
$$

where the $C_{\alpha}^{I}$ are non-covariant constant spinors implicitly defined by the above two equations. The important point now is that $C_{\alpha}^{I} \theta^{\alpha} \delta\left(C_{\beta}^{I} \lambda^{\beta}\right)$ is precisely what Berkovits called $Y_{C^{I}}$ in [11, so that we have recovered his result in our setting. Above, our $C^{I}$ 's were of a very special form, but it was shown in 11 that the expression (2.63) is actually independent of the $C^{I}$ 's. This completes our discussion of the relation between the RNS and pure spinor saturation rules.

In the previous discussion we have exclusively worked within the operator formalism. Let us also briefly discuss the connection to the equivalent path integral approach and its associated zero mode measure. From the above expressions it might naively seem that $\left(\lambda^{+}\right)^{3} \delta\left(\lambda^{+}\right)$is zero. But this expression is meaningless without the measure. The measure associated with (2.60) is the canonical one

$$
\mathrm{d} \gamma^{\prime} \prod_{a b} \mathrm{~d} u_{a b} \prod_{\alpha=1}^{16} \mathrm{~d} \theta^{\alpha}
$$

Under the change of variables from $\gamma^{\prime}$ to $\lambda^{+}=\gamma^{\prime-1 / 2}$ the measure $\mathrm{d} \gamma^{\prime}$ transforms into $\mathrm{d} \lambda^{+} \frac{\mathrm{d} \gamma^{\prime}}{\mathrm{d} \lambda^{+}} \propto \mathrm{d} \lambda^{+}\left(\lambda^{+}\right)^{-3}$, so that what remains after multiplication is $\int \mathrm{d} \lambda^{+} \delta\left(\lambda^{+}\right)$.

\footnotetext{
${ }^{8}$ The expression $\left(\lambda^{+}\right)^{3} \delta\left(\lambda^{+}\right)$, which naively might look like it is zero, is meaningless without the measure. We discuss the measure below; in the operator formalism the measure is implicit.
} 
After implementing this change of variables in (2.64) we obtain the measure appropriate to the saturation rule (2.61). The form of the measure appropriate to the equivalent covariantised saturation rule (2.62) was constructed in [11]. Note also that in the formulation where the pure spinor constraint is relaxed, some aspects of the measure were discussed in [23].

Let us end this subsection with a couple of comments. Notice that with our definitions it is not true that in the ghost sector $T=w_{\alpha} \partial \lambda^{\alpha}=w_{+} \partial \lambda^{+}+\frac{1}{2} w^{a b} \partial \lambda_{a b}$, as can be seen by using (B.3) and (2.5). However, if one redefines $\lambda_{a b} \rightarrow e^{c_{1} s} u_{a b}$ and $w^{a b} \rightarrow e^{-c_{1} s} v^{a b}$, as well as $w_{+} \rightarrow e^{-s}\left(\partial t+c_{2} \partial s+c_{3} v^{a b} u_{a b}\right)$, it is possible to choose the constants $c_{1}, c_{2}$ and $c_{3}$ in such a way that this is true. Also notice that one can define the analogue of the usual RNS picture number for the eleven $\beta \gamma$-type systems: $\left(\beta^{\prime}, \gamma^{\prime}\right),\left(v^{a b}, u_{a b}\right)$. In terms of these, the $Y^{I}$ 's have picture number -1 (and ghost number +1 ). Another point is that it can be checked that the operator saturating the zero modes is BRST closed. In the form (2.61) this is only true for the expression as a whole. On the other hand, for the covariantised expression (2.62) this is essentially true for the $\lambda^{3} \theta^{5}$ and $\prod_{I} Y^{I}$ parts separately [11, as required for the interpretation of the insertion of $\prod_{I} Y^{I}$ as a map between cohomologies [28].

The main result of the recent paper [11] was a prescription for calculating loop amplitudes in the pure spinor formulation, which had been a long standing problem. In the calculation of loop amplitudes in the RNS formulation, insertions roughly of the form $\{Q, \Theta(\beta)\}$ occur, where $\Theta$ is the step-function. If one naively takes this to hold also in the pure spinor formulation, then one obtains (using (2.5) and $\left.\Theta^{\prime}(x)=\delta(x)\right)$

$$
\left\{Q, \Theta\left(v^{a b}\right)\right\}=\frac{1}{2}\left[d^{a b} e^{s}+\frac{1}{2} \epsilon^{a b c d e} d_{c} u_{d e}\right] e^{-s} \delta\left(v^{a b}\right) \propto\left(d \gamma^{a b} \lambda\right) \delta\left(N^{a b}\right) \quad \text { (no sum) }
$$

where in the last step we have used (B.5). Introducing $B_{c d}^{I}=\delta_{c}^{a} \delta_{d}^{b}$, where $I=a b$, we can rewrite the above expression as $B_{c d}^{I}\left(d \gamma^{c d} \lambda\right) \delta\left(B_{e f}^{I} N^{e f}\right)$. Thus we find exactly the $Z_{B^{I}}$ operator defined in [1]. Similarly, using $\beta^{\prime} \propto e^{2 s}(\partial t+2 \partial s)$, one finds

$$
\left\{Q, \Theta\left(\beta^{\prime}\right)\right\} \propto\left[e^{s} d^{+}-\lambda^{a} d_{a}\right] e^{2 s} \delta\left(e^{2 s}(\partial t+2 \partial s)\right)
$$

which is equal to $Q \delta(J)$ modulo normal ordering ambiguities and terms which vanish when multiplied by $\delta\left(u_{a b}\right)$. Thus we find the operator $Z_{J}$ defined in 11]. As we said earlier, it may be possible to make these arguments more precise. Also, related aspects of the loop amplitude prescription, as well as the geometry of the picture changing operators, were recently discussed in [31].

\subsection{Mode Expansions}

In this subsection we collect the mode expansions of the world-sheet fields. Since this is a standard procedure, we shall be rather schematic. The world-sheet bosons $\partial x^{m}, \bar{\partial} x^{m}$, which transform as spacetime vectors, have the standard expansions

$$
\partial x^{m}(z)=-i \sum_{k \in \mathbb{Z}} \frac{\alpha_{k}^{m}}{z^{k+1}}, \quad \bar{\partial} x^{m}(\bar{z})=-i \sum_{k \in \mathbb{Z}} \frac{\widetilde{\alpha}_{k}^{m}}{\bar{z}^{k+1}},
$$


where the spacetime momentum is $p^{m}=\alpha_{0}^{m}=\widetilde{\alpha}_{0}^{m}$, and the commutation relations are as usual $\left[\alpha_{k}^{m}, \alpha_{l}^{n}\right]=k \eta^{m n} \delta_{k+l}$ and similarly for $\widetilde{\alpha}_{k}^{m}$. The world-sheet scalars which transform as spacetime fermions have integral world--sheet conformal dimensions, with mode expansions

$$
\theta^{\alpha}(z)=\sum_{k \in \mathbb{Z}} \frac{\theta_{k}^{\alpha}}{z^{k}}, \quad p_{\alpha}(z)=\sum_{k \in \mathbb{Z}} \frac{p_{\alpha, k}}{z^{k+1}},
$$

and similarly for the right-moving fields. The commutation relations are, as expected, $\left\{p_{\alpha, k}, \theta_{l}^{\beta}\right\}=\delta_{\alpha}^{\beta} \delta_{k+l}$ and similarly for the right-moving sector. The mode expansions of $\partial g$ and $M^{m n}$ are (we suppress the right-moving sector)

$$
\partial g(z)=\sum_{k \in \mathbb{Z}} \frac{g_{k}}{z^{k+1}}, \quad M^{m n}(z)=\sum_{k \in \mathbb{Z}} \frac{M_{k}^{m n}}{z^{k+1}} .
$$

As to the ghost field, $\lambda^{\alpha}$, its expansion is

$$
\lambda^{\alpha}(z)=\sum_{k \in \mathbb{Z}} \frac{\lambda_{k}^{\alpha}}{z^{k}}
$$

Finally, the expansions for $\partial h$ and $N^{m n}$ are completely analogous to the ones in (2.69), and we shall not explicitly write them down.

The commutation relations involving the modes of $\left(M^{m n}, N^{m n}, \partial g, \partial h, \lambda^{\alpha}, \theta^{\alpha}\right)$ can easily be worked out; we have the following non-vanishing commutators

$$
\begin{aligned}
{\left[M_{k}^{r s}, M_{l}^{m n}\right] } & =\eta^{r m} M_{k+l}^{s n}-\eta^{s m} M_{k+l}^{r n}+\eta^{r n} M_{k+l}^{s m}-\eta^{s m} M_{k+l}^{r n}+4 l\left(\eta^{m s} \eta^{n r}-\eta^{m r} \eta^{n s}\right) \delta_{k+l}, \\
{\left[N_{k}^{r s}, N_{l}^{m n}\right] } & =\eta^{r m} N_{k+l}^{s n}-\eta^{s m} N_{k+l}^{r n}+\eta^{r n} N_{k+l}^{s m}-\eta^{s m} N_{k+l}^{r n}-3 l\left(\eta^{m s} \eta^{n r}-\eta^{m r} \eta^{n s}\right) \delta_{k+l}, \\
{\left[N_{k}^{m n}, \lambda_{l}^{\alpha}\right] } & =\frac{1}{2}\left(\gamma^{m n}\right)^{\alpha}{ }_{\beta} \lambda_{k+l}^{\beta}, \quad\left[M_{k}^{m n}, \theta_{l}^{\alpha}\right]=\frac{1}{2}\left(\gamma^{m n}\right)_{\beta}^{\alpha} \theta_{k+l}^{\beta}, \\
{\left[h_{k}, \lambda_{l}^{\alpha}\right] } & =\frac{1}{2} \lambda_{k+l}^{\alpha}, \quad\left[g_{k}, \theta_{l}^{\alpha}\right]=-\frac{1}{4} \theta_{k+l}^{\alpha}, \\
{\left[h_{k}, h_{l}\right] } & =-k \delta_{k+l}, \quad\left[g_{k}, g_{l}\right]=k \delta_{k+l} .
\end{aligned}
$$

Finally, we should also point out that after a conformal transformation to the cylinder, the mode expansions are (in the $(\tau, \sigma)$ cylinder coordinates)

$$
F=\sum_{k} f_{k} e^{-i k(\tau-\sigma)}, \quad \widetilde{F}=\sum_{k} \widetilde{f}_{k} e^{-i k(\tau+\sigma)}
$$

for any fields $F, \widetilde{F}$, irrespective of their conformal weights.

\subsection{Vacua}

We now discuss the vacuum structure of the theory in more detail. Let us first consider a simple toy model comprising one $b c$-system with fields $p$ and $\theta$ and one $\beta \gamma$-system with fields $v$ and $u$. We assume that both systems have conformal weight equal to one. For general weights, one needs to distinguish between the oscillator vacuum and the SL(2) vacuum. However, for the special case of weight one, these 
coincide. We denote the SL(2) invariant oscillator vacuum by $|0\rangle$. This state satisfies $L_{0}|0\rangle=0=L_{ \pm}|0\rangle$. In terms of the oscillator modes in the " $b c$ " sector this state satisfies

$$
p_{k}|0\rangle=0 \text { for } k \geq 0 \text { and } \theta_{k}|0\rangle=0 \text { for } k \geq 1 .
$$

As is well known there is another state with the same energy, namely, $\theta_{0}|0\rangle$. This state has "ghost" number +1 . The notation $|\uparrow\rangle$ is often used for this state whereas the notation $|\downarrow\rangle$ is often used for what we previously called $|0\rangle$. The only nonvanishing matrix element between the degenerate states is $\left\langle 0\left|\theta_{0}\right| 0\right\rangle$ (or using the other notation, $\langle\downarrow \mid \uparrow\rangle)$. In the " $\beta \gamma$ " sector, $|0\rangle$ satisfies

$$
v_{k}|0\rangle=0 \text { for } k \geq 0 \text { and } u_{k}|0\rangle=0 \text { for } k \geq 1 .
$$

As was the case for the $b c$-system, there are other vacuum states. In particular, the state which has a non-vanishing matrix element with $\langle 0|$ is $\delta\left(u_{0}\right)|0\rangle$ (this is usually shown using bosonisation). The state $\delta\left(u_{0}\right)|0\rangle$ has "picture" number -1 . To conclude, there are two natural vacuum states one can define, $|0\rangle$ and $|\Omega\rangle=$ $\theta_{0} \delta\left(u_{0}\right)|0\rangle$, and these states have a non-vanishing inner product as $\langle 0 \mid \Omega\rangle \neq 0$.

Let us now make contact with the saturation rule given in (2.62). This rule can be written as (here we suppress the ' 0 ' zero mode subscripts)

$$
\left\langle 0\left|\left(\lambda \gamma^{m} \theta\right)\left(\lambda \gamma^{n} \theta\right)\left(\lambda \gamma^{p} \theta\right)\left(\theta \gamma_{m n p} \theta\right)\right| \Omega\right\rangle \neq 0
$$

where

$$
|\Omega\rangle=\prod_{I=1}^{11} Y^{I}|0\rangle=\left[\theta^{+} \delta\left(\lambda^{+}\right)\right] \prod_{a b=1}^{10}\left[\theta_{a b} \delta\left(\lambda_{a b}\right)\right]|0\rangle .
$$

Recall that there are eleven $(b, c, \beta, \gamma)$-type quartets of weight one in the $\mathrm{U}(5)$ formulation of the pure spinor superstring: $\left(p_{+}, \theta^{+}, \beta^{\prime}, \gamma^{\prime}\right)$ and the ten $\left(p^{a b}, \theta_{a b}, v^{a b}, u_{a b}\right)$. Given the above discussion we see that the $Y^{I}$ insertions can essentially be understood as a change in vacuum, required to get a non-vanishing matrix element (since $\delta\left(\lambda^{+}\right)$is used rather than $\delta\left(\gamma^{\prime}\right)$ there is a remaining background charge which is cancelled by the explicit additional $\lambda^{3}$ insertion).

\section{Pure Spinor Boundary States in Flat Spacetime}

In this section we discuss the construction of the D-brane boundary state, in the pure spinor superstring. Throughout we restrict ourselves to the case of a flat spacetime background. Some of the results in subsections 3.1 through 3.3, and 3.5 were also obtained in [10, albeit using a slightly different approach. However, the zero mode part of the boundary state was not discussed in [10]. For simplicity we shall concentrate on the D9-brane in the type IIB theory (but a brief discussion of the lower dimensional $\mathrm{D} p$-branes can be found at the end of this section). 


\subsection{The Open String Picture: Sigma Model Boundary Conditions}

We start by considering an open string stretched between two D9-branes in the type IIB string theory. The relation between the $(z, \bar{z})$ coordinates and the $(\tau, \sigma)$ coordinates is

$$
z=e^{i(\tau-\sigma)}, \quad \bar{z}=e^{i(\tau+\sigma)}, \quad \partial_{\tau}=i(z \partial+\bar{z} \bar{\partial}), \quad \partial_{\sigma}=i(-z \partial+\bar{z} \bar{\partial}) .
$$

We are considering an open string with endpoints at $\sigma=0$ and $\sigma=\pi$. To determine the boundary conditions that the open string should obey at its endpoints we consider the variation of the action (2.3). At this point we do not consider the ghost sector; this sector will be included later. The variation of the action gives the equations of motion for the fields, plus the boundary term

$$
\frac{1}{2 \pi} \int d \tau\left[-2 i(z \partial-\bar{z} \bar{\partial}) x_{m} \delta x^{m}-2 i z p_{\alpha} \delta \theta^{\alpha}+2 i \bar{z} \widetilde{p}_{\alpha} \delta \widetilde{\theta}^{\alpha}\right]_{\sigma=0}^{\sigma=\pi},
$$

that needs to be set to zero. Consider $\sigma=0$ corresponding to $z=\bar{z}$. The boundary condition on $x^{m}$ is

$$
\left.\delta x^{m}(z \partial-\bar{z} \bar{\partial}) x_{m}\right|_{\sigma=0}=0 .
$$

This is the usual condition on the bosonic modes of the open string. For a D9-brane we impose Neumann boundary conditions $\left.(z \partial-\bar{z} \bar{\partial}) x^{m}\right|_{\sigma=0}=0$. In terms of the $(\tau, \sigma)$ coordinates this is just $\left.\partial_{\sigma} x^{m}\right|_{\sigma=0}=0$.

Let us now consider the spacetime fermionic sector. It is clear that one needs

$$
\left.\left(z p_{\alpha} \delta \theta^{\alpha}-\bar{z} \widetilde{p}_{\alpha} \delta \widetilde{\theta}^{\alpha}\right)\right|_{\sigma=0}=0 .
$$

The boundary term thus vanishes if one imposes the boundary conditions

$$
\left.\left(z p_{\alpha} \pm \bar{z} \widetilde{p}_{\alpha}\right)\right|_{\sigma=0}=0,\left.\quad\left(\theta^{\alpha} \pm \widetilde{\theta}^{\alpha}\right)\right|_{\sigma=0}=0 .
$$

From the above results (written in terms of the oscillator modes) it follows that the boundary conditions on the supersymmetry generators (2.24) are $^{9}$

$$
\left.\left(z q_{\alpha} \pm \bar{z} \widetilde{q}_{\alpha}\right)\right|_{\sigma=0}=0 .
$$

This implies the well-known fact that the D-brane preserves half the supersymmetries, and justifies the conditions (3.5). It also follows from the above conditions that the fermion Lorentz currents $M^{m n}=-\frac{1}{2} p \gamma^{m n} \theta, \widetilde{M}^{m n}=-\frac{1}{2} \widetilde{p} \gamma^{m n} \widetilde{\theta}$, and the fermion Lorentz scalars $g=\frac{1}{4} p \theta, \widetilde{g}=\frac{1}{4} \widetilde{p} \widetilde{\theta}$, obey the boundary conditions

$$
\left.\left(z M^{m n}-\bar{z} \widetilde{M}^{m n}\right)\right|_{\sigma=0}=0,\left.\quad(z \partial g-\bar{z} \bar{\partial} \widetilde{g})\right|_{\sigma=0}=0 .
$$

\footnotetext{
${ }^{9}$ Observe that in (3.6) we are actually referring to the supersymmetry current and not to the supersymmetry charge. We will return to the standard notation in what follows.
} 
One should not confuse the \pm -ambiguity above with the one appearing in the RNS superstring. Whereas in the RNS case this ambiguity is related to the world-sheet spin structures, the ambiguity here should rather be regarded as a spacetime ambiguity associated to a choice of brane versus anti-brane. That this is so can be understood by the fact that a D-brane breaks half of the target space supersymmetries and, as one can see from (3.6) above, there are two distinct ways to do so: one must be associated to a D-brane and the other to the anti-D-brane. Later, we will indeed see that this sign ambiguity corresponds to objects with the same tension but opposite RR charges, as expected.

\subsection{The Closed String Picture: D-Brane Boundary States}

If one computes the open string one-loop amplitude, with the boundary conditions just found in section 3.1. for open strings ending on a D-brane, one is computing the annulus diagram for the open string. This annulus diagram can equivalently be described in terms of closed string propagation, as is well-known. In order to go from the open string picture to the closed string picture, one essentially needs to do the transformation $\tau \leftrightarrow \sigma$ (see, e.g., [8] for a detailed discussion). In general one also has the option of writing the relevant expressions either in terms of the $(z, \bar{z})$ coordinates on the complex plane, or to make a conformal transformation to the $(\tau, \sigma)$ cylinder coordinates. Under the latter transformation the fields will have fixed transformation properties dictated by their conformal weights, e.g., $\theta^{\alpha} \rightarrow \theta^{\alpha}$ and $\widetilde{\theta}^{\alpha} \rightarrow \widetilde{\theta}^{\alpha}$, whereas $z p_{\alpha} \rightarrow i p_{\alpha}$ and $\bar{z} \widetilde{p}_{\alpha} \rightarrow-i \widetilde{p}_{\alpha}$.

Via the above transformations, one can directly translate the boundary conditions for the open string, obtained in section 3.1. into conditions for the (closed string sector) boundary state $|B\rangle$. Below, all conditions are written in terms of the $(\tau, \sigma)$ variables, and all fields have mode expansions as in (2.72). We find

$$
\begin{gathered}
\left.\partial_{\tau} x^{m}\right|_{\tau=0}|B\rangle=0, \\
\left.\left(p_{\alpha} \mp \widetilde{p}_{\alpha}\right)\right|_{\tau=0}|B\rangle=0,\left.\quad\left(\theta^{\alpha} \pm \widetilde{\theta}^{\alpha}\right)\right|_{\tau=0}|B\rangle=0, \\
\left.\left(M^{m n}+\widetilde{M}^{m n}\right)\right|_{\tau=0}|B\rangle=0,\left.\quad \partial_{\tau}(g+\widetilde{g})\right|_{\tau=0}|B\rangle .
\end{gathered}
$$

In terms of the modes of the fields this translates into

$$
\begin{gathered}
\left(\alpha_{k}^{m}+\widetilde{\alpha}_{-k}^{m}\right)|B\rangle=0, \\
\left(p_{\alpha, k} \mp \widetilde{p}_{\alpha,-k}\right)|B\rangle=0, \quad\left(\theta_{k}^{\alpha} \pm \widetilde{\theta}_{-k}^{\alpha}\right)|B\rangle=0, \\
\left(M_{k}^{m n}+\widetilde{M}_{-k}^{m n}\right)|B\rangle=0, \quad\left(g_{k}+\widetilde{g}_{-k}\right)|B\rangle=0 .
\end{gathered}
$$

for any $k \in \mathbb{Z}$.

We now turn our attention to the ghost sector. It is clear that one could repeat the above analysis for the free $\mathrm{U}(5)$ ghost variables. However, rather than giving 
the details of such an analysis, we shall instead present another approach in what follows (which leads to the same results). First, notice that the boundary state should satisfy

$$
(Q+\widetilde{Q})|B\rangle=0
$$

where the BRST operators were given in (2.20). Consistency of this relation with the boundary condition on $\left(\theta^{\alpha}, \widetilde{\theta}^{\alpha}\right)$ implies that

$$
\left.\left(\lambda^{\alpha} \pm \widetilde{\lambda}^{\alpha}\right)\right|_{\tau=0}|B\rangle=0
$$

which in turn is consistent with the conditions

$$
\left.\left(N^{m n}+\tilde{N}^{m n}\right)\right|_{\tau=0}|B\rangle=0,\left.\quad \partial_{\tau}(h+\widetilde{h})\right|_{\tau=0}|B\rangle=0 .
$$

A further consistency check is that the Lorentz generators in the spacetime fermionic sector and in the ghost sector satisfy the same boundary conditions, as required. Having obtained the boundary conditions, we next turn to the construction of the state $|B\rangle$ satisfying the above conditions.

\subsection{Boundary State: Non-Zero Mode Sector}

Without loss of generality one can write $|B\rangle$ as $e^{W}|B\rangle_{0}$ where the prefactor $e^{W}$ only involves non-zero modes, and $|B\rangle_{0}$ refers to the zero mode part. The part of the $e^{W}$ prefactor involving the bosonic modes of $x^{m}$ is as usual

$$
\exp \left(-\sum_{k=1}^{\infty} \frac{1}{k} \alpha_{m,-k} \widetilde{\alpha}_{-k}^{m}\right) .
$$

Similarly, the corresponding $p \theta$ prefactor is

$$
\exp \left(\mp \sum_{k=1}^{\infty}\left[p_{\alpha,-k} \widetilde{\theta}_{-k}^{\alpha}+\widetilde{p}_{\alpha,-k} \theta_{-k}^{\alpha}\right]\right) .
$$

The ghost part of the prefactor can, e.g., be written in terms in terms of the $w \lambda$ variables as (we could also have written it in terms of the free $\left(\beta^{\prime}, \gamma^{\prime}, v^{a b}, u_{a b}\right)$ variables and their right-moving cousins)

$$
\exp \left( \pm \sum_{k=1}^{\infty}\left[w_{\alpha,-k} \widetilde{\lambda}_{-k}^{\alpha}+\widetilde{w}_{\alpha,-k} \lambda_{-k}^{\alpha}\right]\right)
$$

It is important to observe that although this expression is seemingly not manifestly covariant (because of the presence of $w_{\alpha}, \widetilde{w}_{\alpha}$ ), the boundary conditions it implies are indeed covariant. Notice that when (3.19) is written in $\mathrm{U}(5)$ components, only the canonically conjugate variables $\left(w_{+}, \lambda^{+}\right),\left(w^{a b}, \lambda_{a b}\right)$ (and their right-moving counterparts) appear, since $w_{a}=0=\widetilde{w}_{a}(c f$. appendix $(\mathrm{B})$. It is easy to see that this is not in conflict with the boundary condition $\left(\lambda^{a} \pm \tilde{\lambda}^{a}\right)|B\rangle=0$ because of the composite 
nature of $\lambda^{a}, \widetilde{\lambda}^{a}$ implied by the pure spinor constraint. Let us also mention that the construction of the cross-cap state $|C\rangle$ is completely analogous, the only difference being that one finds extra $(-1)^{k}$ insertions in the sums in the above expressions.

In this paper the above explicit expressions for the non-zero mode part of the boundary state will play a marginal role, so we shall not discuss them any further in this work. Instead we turn to the much harder problem of determining $|B\rangle_{0}$ - the zero mode part of the boundary state.

\subsection{Boundary State: Zero Mode Sector}

As discussed above, the boundary state $|B\rangle$ contains two parts: a part containing the non-zero modes and a part containing only zero modes. There are several different possible routes one could take to obtain the zero mode part of the covariant boundary state in the pure spinor superstring. Some possibilities are:

- Start from the known expression in the RNS superstring and try to apply the map in [13] in order to obtain a result in terms of the pure spinor variables.

- Start from RNS, written in its U(5) version 30, and derive the boundary states directly in terms of these variables, then translate the result so obtained into the pure spinor superstring.

- Start directly with the pure spinor variables, alongside with the associated boundary conditions, and try to derive the boundary state.

- Use known information about the pure spinor massless vertex operators (the zero mode cohomology) in order to try to obtain information about the zero mode part of the boundary state.

Below we will comment on each of these approaches. As above, we restrict ourselves to the D9-brane in type IIB; the more general case will be discussed later. Also, in order not to clutter the formulæ, we shall not explicitly write the index ' 0 ' on the various variables to indicate that, in this subsection, we are dealing exclusively with the zero modes. From the previous discussion it follows that we want the zero mode part of the boundary state to satisfy the conditions

$$
\begin{aligned}
& \left(\theta^{\alpha} \pm \widetilde{\theta}^{\alpha}\right)|B\rangle_{0}=0, \quad\left(p_{\alpha} \mp \widetilde{p}_{\alpha}\right)|B\rangle_{0}=0, \quad\left(\lambda^{\alpha} \pm \widetilde{\lambda}^{\alpha}\right)|B\rangle_{0}=0, \\
& (Q+\widetilde{Q})|B\rangle_{0}=0, \quad\left(L^{m n}+\widetilde{L}^{m n}\right)|B\rangle_{0}=0, \quad\left(q_{\alpha} \mp \widetilde{q}_{\alpha}\right)|B\rangle_{0}=0 \text {. }
\end{aligned}
$$

The first condition, together with the last, implies that the $\theta, \widetilde{\theta}$ dependence of the zero mode part of $|B\rangle$ has to be $|B\rangle_{0}^{\theta, \widetilde{\theta}}=\prod_{\alpha=1}^{16}\left(\theta^{\alpha} \pm \widetilde{\theta}^{\alpha}\right)|0\rangle$, where $|0\rangle$ is annihilated by $p_{\alpha}$ and $\widetilde{p}_{\alpha}$ (additional factors of $\theta, \widetilde{\theta}$ are ruled out by the fact that we want precisely half the supersymmetry generators to annihilate the boundary state).

In the ghost sector the situation is more involved. It is clear that we want a factor $\delta\left(\lambda^{\alpha} \pm \widetilde{\lambda}^{\alpha}\right)$ for each independent component of $\lambda, \widetilde{\lambda}$. However, since the $\lambda, \widetilde{\lambda}$ variables are bosonic an additional multiplicative function of $\lambda, \widetilde{\lambda}$ might also be allowed and 
it is not immediately obvious how to choose this function. The approach we shall now take to determine the ghost part of the boundary state is based on the free $\mathrm{U}(5)$ variables; at the end we will check that all boundary conditions are satisfied.

In terms of the $\mathrm{U}(5)$ ghost variables (which we recall comprise eleven $\beta \gamma$-systems, all of conformal weight one) the natural candidate for the zero mode part of the boundary state is $|B\rangle_{0}^{\gamma^{\prime}, u ; \tilde{\gamma}^{\prime}, \widetilde{u}}=\delta\left(\gamma^{\prime}-\widetilde{\gamma}^{\prime}\right) \prod_{a b} \delta\left(u_{a b} \pm \widetilde{u}_{a b}\right)|0\rangle$, where $|0\rangle$ has picture number 0 and is annihilated by $\beta^{\prime}, \widetilde{\beta}^{\prime}, v^{a b}$ and $\widetilde{v}^{a b}$. Strictly speaking there are some sign ambiguities here. We have chosen to fix the signs so that Lorentz covariance is recovered at the end (see the later discussion). Recalling that $\gamma^{\prime}=\left(\lambda^{+}\right)^{-2}$ (and similarly for $\widetilde{\gamma^{\prime}}$ ), we see that $\gamma^{\prime}=\widetilde{\gamma^{\prime}}$ implies $\lambda^{+}=\mp \tilde{\lambda}^{+}$. The transformation of $\delta\left(\gamma-\gamma^{\prime}\right)$ to the $\lambda^{+}, \widetilde{\lambda}^{+}$variables gives $\delta\left(\gamma-\gamma^{\prime}\right) \propto\left(\lambda^{+}\right)^{k}\left(\tilde{\lambda}^{+}\right)^{3-k} \delta\left(\lambda^{+} \pm \widetilde{\lambda}^{+}\right)$, where the integer $k$, with $0 \leq k \leq 3$, is arbitrary. This arbitrariness arises from the presence of the delta function (there is a similar ambiguity in the radial part of the usual three dimensional delta function expressed in spherical coordinates). In the path integral approach the integration is performed using the closed string measures $\int \mathrm{d} \lambda^{+}\left(\lambda^{+}\right)^{-3}$ and $\int \mathrm{d} \widetilde{\lambda}^{+}\left(\widetilde{\lambda}^{+}\right)^{-3}$; this shows that all $k$ give identical results.

Combining the above results we (schematically) obtain

$$
|B\rangle_{0} \propto \sum_{n=0}^{5}( \pm)^{n}\left(\lambda^{+}\right)^{k_{n}}\left(\widetilde{\lambda}^{+}\right)^{3-k_{n}} \theta^{n} \widetilde{\theta}^{5-n} \prod_{I=1}^{11} Y^{I}(\theta \pm \widetilde{\theta}, \lambda \pm \widetilde{\lambda})|0\rangle
$$

where, as in section 2.2, we have re-written the delta functions involving $\theta^{+}, \theta_{a b}$, $\lambda^{+}$, and $\lambda_{a b}$ in terms of $Y^{I}(\theta, \lambda)=C_{\alpha}^{I} \theta^{\alpha} \delta\left(C_{\alpha}^{I} \lambda^{\alpha}\right)$ for certain constant spinors $C_{\alpha}^{I}$. The remaining $\theta^{a}, \widetilde{\theta}^{a}$ variables have been written using a short-hand notation, e.g., $\theta^{2} \widetilde{\theta}^{3}=\epsilon_{a b c d e} \theta^{a} \theta^{b} \widetilde{\theta}^{c} \widetilde{\theta}^{d} \widetilde{\theta}^{e}$ and so on. The basic boundary conditions for the free fields discussed above can be used to show that the boundary state (3.21) satisfies the correct boundary conditions also for the composite fields $Q, q_{\alpha}, M^{m n}, \partial g, N^{m n}$ and $\partial h$, by using their explicit expressions in terms of the free fields.

We will now argue that, without changing the physics, one can replace the $\left(\lambda^{+}\right)^{k_{n}}\left(\widetilde{\lambda}^{+}\right)^{3-k_{n}} \theta^{n} \widetilde{\theta}^{5-n}$ pieces in the boundary state by manifestly covariant expressions, for instance one may replace $\lambda^{+}\left(\widetilde{\lambda}^{+}\right)^{2} \theta \widetilde{\theta}^{4} \rightarrow\left(\lambda \gamma^{m} \theta\right)\left(\widetilde{\lambda} \gamma^{n} \widetilde{\theta}\right)\left(\widetilde{\lambda} \gamma^{p} \widetilde{\theta}\right)\left(\widetilde{\theta} \gamma_{m n p} \widetilde{\theta}\right)$. One direct argument is to note that the presence of the $\delta\left(\lambda^{\alpha}-\tilde{\lambda}^{\alpha}\right)$ 's in the $Y^{I}$ 's means that in the covariant expressions we can replace, e.g., all $\widetilde{\lambda}^{\alpha}$ s sy $\lambda^{\alpha}$ s. The remaining $\theta, \widetilde{\theta}$ dependence is then via $\theta^{\alpha}-\widetilde{\theta}^{\alpha}$, but the fermionic delta functions in the $Y^{I}$ 's then put the ${ }^{+}$and ${ }_{a b}$ components to zero. Finally, one notes that there is no way to form a non-vanishing scalar out of a product of five $(\theta-\widetilde{\theta})^{a}$ 's as well as $\epsilon_{a b c d e}$ and $\lambda^{+}$, which also involves $\lambda^{a}$ and/or $\lambda_{a b}$. Thus only the $\lambda^{+}$dependence survives and we are back to the above expression (3.21).

An indirect but perhaps more transparent argument is to note that in calculating correlation functions (and as we shall later see, when calculating scattering amplitudes) one also needs to insert $\prod_{I=1}^{11} Y^{I}(\theta \mp \widetilde{\theta}, \lambda \mp \widetilde{\lambda})$ to saturate the zero modes. 
Using the fact that

$$
\prod_{I=1}^{11} Y^{I}(\theta \mp \widetilde{\theta}, \lambda \mp \widetilde{\lambda}) Y^{I}(\theta \pm \widetilde{\theta}, \lambda \pm \widetilde{\lambda}) \propto \prod_{I=1}^{11} Y^{I}(\theta, \lambda) Y^{I}(\widetilde{\theta}, \widetilde{\lambda}) \equiv \prod_{I=1}^{11} Y^{I} \widetilde{Y}^{I}
$$

one realises that the presence of the delta functions in the $Y^{I}$ and $\widetilde{Y}^{I}$ 's means that in the covariant expressions only the terms present in (3.21) survive.

There still remains the ambiguity in choosing the $k_{n}$ 's, though. Although this ambiguity does not appear to have any direct physical meaning there is a certain choice which is quite convenient. To describe this choice we recall that the left- and right-moving parts of the vertex operators for the massless states involve certain basic constituent operators. These operators essentially coincide with the zero mode cohomology (which was explicitly worked out in 32; see also 33, 34, 28]) and are summarised in the table below.

\begin{tabular}{|c|c|}
\hline$(p, q)$ & $U_{p, q}$ \\
\hline$(0,0)$ & 1 \\
$(1,0) *$ & $\lambda^{\alpha}$ \\
$(1,1)$ & $\left(\lambda \gamma^{m} \theta\right)$ \\
$(1,2)$ & $\left(\lambda \gamma^{m} \theta\right)\left(\theta \gamma_{m}\right)_{\alpha}$ \\
$(2,3)$ & $\left(\lambda \gamma^{m} \theta\right)\left(\lambda \gamma^{n} \theta\right)\left(\gamma_{m n} \theta\right)^{\alpha}$ \\
$(2,4)$ & $\left(\lambda \gamma^{m} \theta\right)\left(\lambda \gamma^{n} \theta\right)\left(\theta \gamma_{m n p} \theta\right)$ \\
$(2,5) *$ & $\left(\gamma^{m} \theta\right)_{\alpha}\left(\lambda \gamma^{n} \theta\right)\left(\lambda \gamma^{p} \theta\right)\left(\theta \gamma_{m n p} \theta\right)$ \\
$(3,5)$ & $\left(\lambda \gamma^{m} \theta\right)\left(\lambda \gamma^{n} \theta\right)\left(\lambda \gamma^{p} \theta\right)\left(\theta \gamma_{m n p} \theta\right)$ \\
\hline
\end{tabular}

Table 2: Basic "massless" operators. The entries marked with * are not in the BRST cohomology.

These operators are normalised according to

$$
\left\langle U_{p, q} U_{r, s}\right\rangle=c \delta_{3-p-r} \delta_{5-q-s},
$$

where $c$ is a constant which depends on conventions and which we shall take to be equal to 1 . In the above formula we have suppressed the index contractions, as well as the extra $Y^{I}$ insertions needed to get a non-vanishing answer. Guided by the properties of the $U_{p, q}$ 's we fix the $k_{n}$ ambiguity so that the "covariant" part of the zero mode boundary state contains only operators of this type. By comparison with the situation in RNS we also require the terms in the "covariant" part of the zero mode part of the boundary state to have $(\lambda, \widetilde{\lambda})$ ghost numbers $(1,2)$ or $(2,1)$. This choice will be further discussed below. Our way of fixing the $k_{n}$ ambiguity implies that although $Q+\widetilde{Q}$ of course annihilates the full boundary state by construction, it now also almost annihilates the "covariant" part (i.e., the part without the $Y^{I}$ 's) separately without using the delta functions. We say almost, because for the states with five $\theta$ 's (five $\widetilde{\theta}^{\prime}$ 's) we choose $U_{2,5} \widetilde{U}_{1,0}\left(U_{1,0} \widetilde{U}_{2,5}\right)$ rather than $U_{3,5}\left(\widetilde{U}_{3,5}\right)$. 
From these requirements it follows that the zero mode part of the boundary state takes the final form

$$
|B\rangle_{0}=\sum_{i=0}^{2}\left[( \pm)^{i} U_{2, i+3} \widetilde{U}_{1,2-i}+( \pm)^{i+1} U_{1,2-i} \widetilde{U}_{2, i+3}\right] \prod_{I=1}^{11} Y^{I}(\theta \pm \widetilde{\theta}, \lambda \pm \widetilde{\lambda})|0\rangle
$$

where $U_{p, q}\left(\widetilde{U}_{p, q}\right)$ is a state in table 2 of the schematic form $\lambda^{p} \theta^{q}\left(\widetilde{\lambda}^{p} \widetilde{\theta}^{q}\right)$. In this expression indices have been suppressed (index contractions are the natural ones).

Equation (3.24) is our final result for the zero mode part of the boundary state. It was constructed to satisfy the boundary conditions (3.20). From this requirement we showed that it could be written as a part containing $Y^{I}$ 's, times another part which, by the arguments given above, could be written in a manifestly covariant manner and which we chose to express in terms of the operators in table 2. Suppressing the $Y^{I}$ insertions by defining $|Z\rangle=\prod_{I=1}^{11} Y^{I}(\theta \pm \widetilde{\theta}, \lambda \pm \widetilde{\lambda})|0\rangle$, the zero mode part of the boundary state takes the form

$$
|B\rangle_{0}=\sum_{i=0}^{2}\left[( \pm)^{i} U_{2, i+3} \widetilde{U}_{1,2-i}+( \pm)^{i+1} U_{1,2-i} \widetilde{U}_{2, i+3}\right]|Z\rangle
$$

where $U(\widetilde{U})$ are the right- (left-) moving "open string" vertex operators for the massless fields given in table 2. Such a form was expected on general grounds, as well as from comparison with RNS, where similar results hold 6] (see also the discussion below). The main rationale behind our choice for fixing the $k_{n}$ ambiguities is that this choice is such that, when performing calculations, the $Y^{I}, \widetilde{Y}^{I}$ insertions largely decouple and one can essentially ignore them and effectively use the above form (3.25), together with the original saturation rule (2.45). However, one must always keep in mind that the $Y^{I}$ insertions are crucial to make sure that $|B\rangle_{0}$ satisfies the correct boundary conditions. For other choices of fixing the ambiguity one would have to use the delta functions in the $Y^{I}$ 's to replace $\lambda^{\prime}$ 's by $\tilde{\lambda}^{\prime}$ 's at certain intermediate stages of the calculations. We should also point out that there may well be a more physical way to understand our expression, perhaps starting from the observation that the covariant piece in the zero mode boundary state (3.25) has total ghost charge three and total spacetime fermion charge five (by total we mean sum of left and right), as does the pure spinor saturation rule.

As a final comment, and in order to gather some additional circumstantial support for our proposal, we now briefly (and at a very heuristic level) discuss the relation to the RNS expressions. In the RR sector the zero mode part of the RNS boundary state involves expressions of the form

$$
\begin{aligned}
& e^{2 \sigma} e^{\widetilde{\sigma}} e^{-1 / 2 \phi} e^{-3 / 2 \widetilde{\phi}} S_{\alpha} \widetilde{S}^{\alpha}=e^{2 \sigma} e^{-1 / 2 \phi} e^{\widetilde{\sigma}} e^{-3 / 2 \widetilde{\phi}} S_{+} \widetilde{S}^{+}+\cdots \quad \propto\left(\lambda^{+}\right)^{2} \widetilde{\lambda}^{+} \theta^{5}+\cdots, \\
& e^{\sigma} e^{2 \widetilde{\sigma}} e^{-1 / 2 \phi} e^{-3 / 2 \widetilde{\phi}} S_{\alpha} \widetilde{S}^{\alpha}=\frac{1}{2} e^{\sigma} e^{-1 / 2 \phi} e^{\widetilde{2 \sigma}} e^{-3 / 2 \widetilde{\phi}} S^{a b} \widetilde{S}_{a b}+\cdots \propto \lambda^{+}\left(\widetilde{\lambda}^{+}\right)^{2} \theta^{2} \widetilde{\theta}^{3}+\cdots, \\
& e^{\sigma} e^{2 \widetilde{\sigma}} e^{-3 / 2 \phi} e^{-1 / 2 \widetilde{\phi}} S^{\alpha} \widetilde{S}_{\alpha}=e^{\sigma} e^{-3 / 2 \phi} e^{2 \widetilde{\sigma}} e^{-1 / 2 \widetilde{\phi}} S^{+} \widetilde{S}_{+}+\cdots \quad \propto \lambda^{+}\left(\widetilde{\lambda}^{+}\right)^{2} \widetilde{\theta}^{5}+\cdots, \\
& e^{2 \sigma} e^{\widetilde{\sigma}} e^{-3 / 2 \phi} e^{-1 / 2 \widetilde{\phi}} S^{\alpha} \widetilde{S}_{\alpha}=\frac{1}{2} e^{2 \sigma} e^{-3 / 2 \phi} e^{\widetilde{\sigma}} e^{-1 / 2 \widetilde{\phi}} S_{a b} \widetilde{S}^{a b}+\cdots \propto\left(\lambda^{+}\right)^{2} \widetilde{\lambda}^{+} \theta^{3} \widetilde{\theta}^{2}+\cdots,
\end{aligned}
$$


where we used a short-hand notation, e.g., $\theta^{2} \widetilde{\theta}^{3}=\epsilon_{a b c d e} \theta^{a} \theta^{b} \widetilde{\theta}^{c} \widetilde{\theta}^{d} \widetilde{\theta}^{e}$. Thus one can surmise the appearance of four of the six terms in (3.24).

In the NSNS sector things are more subtle since there one has to distinguish between the zero mode sector, and the part of the boundary state which couples to the massless fields. In the pure spinor case there is no such distinction, so what one should try to map to the pure spinor zero mode boundary state (which is also the part which couples to the massless sector) is the part of the NSNS boundary state which couples to the massless modes, namely

$$
|B\rangle_{m^{2}=0, \mathrm{NSNS}} \propto\left[e^{\sigma} e^{2 \widetilde{\sigma}}+e^{\sigma} e^{2 \widetilde{\sigma}}\right] e^{-\phi} e^{-\widetilde{\phi}} \Psi^{m} \widetilde{\Psi}_{m}|0\rangle_{\mathrm{NS}}
$$

Using

$$
\begin{aligned}
& e^{\sigma} e^{2 \widetilde{\sigma}} e^{-\phi} e^{-\widetilde{\phi}} \Psi^{m} \widetilde{\Psi}_{m}=e^{\sigma} e^{-\phi} e^{2 \widetilde{\sigma}} e^{-\widetilde{\phi}} \psi^{a} \widetilde{\psi}_{a}+\cdots \propto \lambda^{+}\left(\widetilde{\lambda}^{+}\right)^{2} \theta \widetilde{\theta}^{4}+\cdots \\
& e^{2 \sigma} e^{\widetilde{\sigma}} e^{-\phi} e^{-\widetilde{\phi}} \Psi^{m} \widetilde{\Psi}_{m}=e^{2 \sigma} e^{-\phi} e^{\widetilde{\sigma}} e^{-\widetilde{\phi}} \psi_{a} \widetilde{\psi}^{a}+\cdots \propto\left(\lambda^{+}\right)^{2} \widetilde{\lambda}^{+} \theta^{4} \widetilde{\theta}+\cdots
\end{aligned}
$$

we find evidence for the remaining two terms in (3.24).

Of course we do not pretend to have actually derived any of the terms in (3.24) from the RNS expressions. Nevertheless, the above relations are an indication that terms of this form are expected to occur from a more careful treatment. It may be possible to make this more precise using the map in [13], although we have not tried to do so. We expect the map between the RNS and pure spinor boundary states to be rather subtle because of the different large/small Hilbert spaces involved in the two cases ( $c f$. the discussion in section 2.2). Another likely source of complications is that the zero mode part of the RR boundary state is really more subtle than we pretended above, see, e.g., [35, 36] for further discussions.

\subsection{The Generalisation to Lower Dimensional $\mathrm{D} p$-Branes}

As is well known the lower dimensional D-branes are obtained by imposing Dirichlet instead of Neumann conditions in some of the spacetime directions. In the general case, the boundary condition involving $x^{m}$ is (here $\partial_{ \pm}=\partial_{\tau} \pm \partial_{\sigma}$ )

$$
\left.\left(\partial_{-} x^{m}+R_{n}^{m} \partial_{+} x^{n}\right)\right|_{\tau=0}|B\rangle=0
$$

where $R$ is the matrix

$$
R_{n}^{m}=\left(\begin{array}{cc}
\delta^{a}{ }_{b} & 0 \\
0 & -\delta^{i}{ }_{j}
\end{array}\right),
$$

with $a, b=0, \ldots, p$ and $i, j=p+1, \ldots, 9$. Our ansatz for the boundary conditions in the spacetime fermionic sector is (for simplicity we only consider the type IIB theory; the type IIA case is similar)

$$
\left.\left(p_{\alpha} \mp R_{\alpha}{ }^{\beta} \widetilde{p}_{\beta}\right)\right|_{\tau=0}|B\rangle=0,\left.\quad\left(\theta^{\alpha} \pm R_{\beta}^{\alpha} \widetilde{\theta}^{\beta}\right)\right|_{\tau=0}|B\rangle=0 .
$$


At this point the matrices $R_{\alpha}{ }^{\beta}$ and $R^{\alpha}{ }_{\beta}$ should be seen as independent, but consistency of the above two equations gives the restriction $R_{\alpha}^{\gamma} R_{\gamma}{ }^{\beta}=\delta_{\alpha}^{\beta}$. Since this is just a relation between matrices, one also has $R_{\alpha}^{\gamma} R_{\gamma}^{\beta}=\delta_{\alpha}^{\beta}$.

Further restrictions follow from the boundary conditions on the supersymmetry and Lorentz currents:

$$
\left.\left(q_{\alpha} \mp R_{\alpha}^{\beta} \widetilde{q}_{\beta}\right)\right|_{\tau=0}|B\rangle=0,\left.\quad\left(M^{m n}+R_{r}^{m} R_{s}^{n} \widetilde{M}^{r s}\right)\right|_{\tau=0}|B\rangle=0 .
$$

These conditions imply the relations

$$
\gamma_{\alpha \beta}^{m} R_{\gamma}^{\beta} R_{m}^{n}=R_{\alpha}{ }^{\delta} \gamma_{\delta \gamma}^{n}
$$

and

$$
\gamma_{\alpha \beta}^{m} R_{[\sigma}^{\beta} R_{\rho]}^{\eta}\left(\gamma_{m}\right)_{\eta \delta} R_{\kappa}^{\delta}=R_{\alpha}^{\beta} \gamma_{\beta[\sigma}^{n}\left(\gamma_{n}\right)_{\rho] \kappa},
$$

as well as

$$
R_{\alpha}{ }^{\eta}\left(\gamma^{m n}\right)_{\beta}^{\alpha} R_{\delta}^{\beta}=R_{r}^{m} R_{s}^{n}\left(\gamma^{r s}\right)_{\delta}^{\eta} .
$$

The solution to the above conditions is (for type IIB with $p$ odd)

$$
R_{\alpha}{ }^{\beta}=\left(\gamma_{p+1 \ldots 9}\right)_{\alpha}{ }^{\beta}=(-1)^{\left[\frac{p-1}{2}\right]}\left(\gamma_{p+1 \ldots 9}\right)_{\beta}^{\alpha}, \quad R_{\alpha}^{\beta}=\left(\gamma_{p+1 \ldots 9}\right)_{\alpha}^{\beta} .
$$

For $p=9$ both $R_{\alpha}{ }^{\beta}$ and $R^{\alpha}{ }_{\beta}$ are equal to $\delta_{\alpha}^{\beta}$. One can check that the matrices (3.34) satisfy all of the above restrictions. In the ghost sector the boundary conditions are

$$
\left.\left(\lambda^{\alpha} \pm R^{\alpha}{ }_{\beta} \widetilde{\lambda}^{\beta}\right)\right|_{\tau=0}|B\rangle=0,\left.\quad\left(N^{m n}+R_{r}^{m} R_{s}^{n} \tilde{N}^{r s}\right)\right|_{\tau=0}|B\rangle=0
$$

which is consistent with $\left.(Q+\widetilde{Q})\right|_{\tau=0}|B\rangle=0$.

The boundary conditions for the modes of the fields can easily be worked out, for instance one has (note the extra condition for the center of mass in the directions transverse to the brane)

$$
\begin{gathered}
\left(\alpha_{k}^{m}+R_{n}^{m} \widetilde{\alpha}_{-k}^{n}\right)|B\rangle=0, \quad X^{i}|B\rangle=y^{i}|B\rangle, i=p+1, \ldots, 9, \\
\left(p_{\alpha, k} \mp R_{\alpha}{ }^{\beta} \widetilde{p}_{\beta,-k}\right)|B\rangle=0, \quad\left(\theta_{k}^{\alpha} \pm R^{\alpha}{ }_{\beta} \theta^{\beta}-k\right)|B\rangle=0, \\
\left(L_{k}^{m n}+R^{m}{ }_{\rho} R^{n}{ }_{s} \widetilde{L}_{-k}^{r s}\right)|B\rangle=0,
\end{gathered}
$$

for any $k \in \mathbb{Z}$. The part of the prefactor in the boundary state involving the bosonic modes becomes

$$
\delta^{9-p}\left(X^{i}-y^{i}\right) \exp \left(-\sum_{k=1}^{\infty} \frac{1}{k} \alpha_{-k}^{m} R_{m n} \widetilde{\alpha}_{-k}^{n}\right) \text {. }
$$


The prefactor involving the non-zero mode in the $p \theta$ sector is

$$
\exp \left(\mp \sum_{k=1}^{\infty}\left[p_{\alpha,-k} R_{\beta}^{\alpha} \widetilde{\theta}_{-k}-\theta_{-k}^{\alpha} R_{\alpha}{ }^{\beta} \widetilde{p}_{\beta,-k}\right]\right),
$$

with a similar modification for the part involving the non-zero modes for the ghost fields. Finally, the zero mode part (3.25) gets modified to

$$
\begin{aligned}
|B\rangle_{0}= & {\left[U_{2,3}^{\alpha} R_{\alpha}{ }^{\beta} \widetilde{U}_{1,2 ; \beta} \pm U_{2,4}^{m} R_{m n} \widetilde{U}_{1,1}^{n}+U_{2,5 ; \alpha} R^{\alpha}{ }_{\beta} \widetilde{U}_{1,0}^{\beta}\right.} \\
& \left. \pm U_{1,2 ; \alpha} R^{\alpha}{ }_{\beta} \widetilde{U}_{2,3}^{\beta}+U_{1,1}^{m} R_{m n} \widetilde{U}_{2,4}^{n} \pm U_{1,0}^{\alpha} R_{\alpha}{ }^{\beta} \widetilde{U}_{2,5 ; \beta}\right]|Z\rangle
\end{aligned}
$$

where $|Z\rangle$ is now also modified to reflect the new boundary conditions.

\section{Applications and Checks}

Although we have presented concise arguments in support of our expression for the boundary state in the pure spinor superstring, it is very important to check that results obtained from earlier calculations in the RNS superstring are recovered in this new formalism. One such test is to calculate scattering amplitudes in the presence of a D-brane and check that the earlier known results are correctly reproduced. As we shall see this requires some further work. Another test of our expression is to extend it to include the coupling to the gauge field living on the D-brane world-volume and to check that the resulting D-brane low-energy effective action is in agreement with the well-known results.

\subsection{Calculating Scattering Amplitudes}

In the RNS approach, tree level scattering amplitudes in the presence of a D-brane can be calculated using (in this subsection we will only consider scattering amplitudes involving the bulk modes)

$$
\left\langle\mathcal{U}\left|\left[c_{0}-\widetilde{c}_{0}\right] \prod_{i=1}^{n-1} \int_{\left|z_{i}\right|>1} \mathrm{~d}^{2} z_{i} V_{i}\left(z_{i}, \bar{z}_{i}\right)\right| B\right\rangle .
$$

Here $\langle\mathcal{U}| \equiv\langle 0| \mathcal{U}(0,0)$, where the unintegrated vertex operator $\mathcal{U}(z, \bar{z})=U(z) \widetilde{U}(\bar{z})$ is assumed to have ghost number $(+1,+1)$ and to be in the appropriate picture to give a non-zero answer for the amplitude. The integrated vertex operators $V_{i}$ are assumed to have zero ghost- and picture-numbers. See, e.g., 37 for a discussion about how expressions such as the one above arise. The explicit $c$-ghost zero mode insertion is needed in order to get a non-zero answer. In the RNS case one does not have to be precise about the $c$-ghost insertions since the ghosts are Lorentz scalars and decouple completely. However, in the pure spinor formalism the $\lambda^{\alpha}$ ghosts have a Lorentz spinor index and do not decouple, so for later reference it is helpful to keep the ghosts in the above expression. 
In generalising the above expression to the pure spinor case, one possible approach is to try to find an analogue for the $c_{0}$-ghost insertion. That additional zero mode insertions are needed also in the pure spinor case is clear from our expression for the boundary state (3.25), along with the ghost charges and number of $\theta$ 's in the unintegrated vertex operators, see, e.g., (2.32), (2.40). One proposal for the required zero mode insertion is (as in RNS we will use the notation $c_{0}$; hopefully this will not lead to confusion)

$$
c_{0}=\frac{1}{2} \oint\left(\lambda \gamma^{m} \theta\right)\left(\theta \gamma_{m n p} \theta\right) L^{n p}
$$

and analogously for $\widetilde{c}_{0}$. Here we have written an expression which, after performing the integral, involves all the modes of $\theta, \lambda, \ldots$, but in practice we only need the part involving purely the zero modes. Some evidence for the choice (4.2) can be obtained by noting that in RNS (using $\mathrm{U}(5)$ notation)

$$
\epsilon_{a b c d e} \oint\left(\lambda^{+} \theta^{c} \theta^{d} \theta^{e}\left(\psi^{a} \psi^{b}\right)\right) \propto \oint\left(c\left(\psi^{a} \psi^{b}\right)\left(\psi_{a} \psi_{b}\right)\right) \propto \oint \mathrm{d} z \frac{c}{z^{2}}+\cdots=c_{0}+\cdots
$$

Since one expects that $\psi^{a} \psi^{b}$ gets replaced by $L^{a b}$ in the pure spinor formalism and since one has $\epsilon_{a b c d e} \lambda^{+} \theta^{c} \theta^{d} \theta^{e} \propto\left(\lambda \gamma^{m} \theta\right)\left(\theta \gamma_{m a b} \theta\right)+\cdots$, the covariant expression suggested in (4.2) seems like a reasonable guess.

With some work, it can be shown that the action of the $c_{0}$ operator on the zero mode states listed in table 2 is such that

$$
c_{0}: U_{1, i} \rightarrow-(-)^{\delta_{i 0}} U_{2, i+3}, \quad i=0,1,2,
$$

i.e., it takes a vertex operator with ghost number 1 into a vertex operator with ghost number 2. Notice that in the present case what we call $c_{0}$ arises from integration of a conformal weight one field, unlike the $c_{0}$-ghost in the RNS case. The rather remarkable result (4.4) can be used to rewrite the zero mode part of the boundary state (3.25) as (here we have also included an overall normalisation factor)

$$
\begin{aligned}
|B\rangle_{0} & = \pm T_{9}\left(c_{0} \mp \widetilde{c_{0}}\right)\left[-U_{1,1} \widetilde{U}_{1,1} \pm\left(U_{1,0} \widetilde{U}_{1,2}-\widetilde{U}_{1,0} U_{1,2}\right)\right]|Z\rangle \\
& =T_{9} \sum_{i=0}^{2}\left[( \pm)^{i} U_{2, i+3} \widetilde{U}_{1,2-i}+( \pm)^{i+1} U_{1,2-i} \widetilde{U}_{2, i+3}\right]|Z\rangle
\end{aligned}
$$

Here the factor $\left(c_{0} \mp \widetilde{c_{0}}\right)$ is assumed to act only on the $U \widetilde{U}$ part (and not on $|Z\rangle$ ). Thus we see that, apart from the $\left(c_{0} \mp \widetilde{c}_{0}\right)$ factor, the zero mode boundary state is simply the sum of a "graviton"-type and a " $C$-field"-type vertex operator, $c f$. (2.32) and (2.40). This result is similar to the situation occurring in the RNS superstring, where an analogous result holds.

The above considerations lead to our proposal for the calculation of scattering amplitudes in the presence of a D-brane, in the pure spinor superstring:

$$
\left\langle\mathcal{U}\left|\left(c_{0} \pm \widetilde{c_{0}}\right) \prod_{i=1}^{n-1} \int_{\left|z_{i}\right|>1} \mathrm{~d}^{2} z_{i} V_{i}\left(z_{i}, \bar{z}_{i}\right)\right| B\right\rangle
$$


with

$$
\langle\mathcal{U}|=\langle Z| U \widetilde{U},
$$

where $U \widetilde{U}$ is taken as a ghost number $(-1,-1)$ vertex operator and where by definition $\langle Z|=\langle 0| \prod_{I=1}^{11} Y^{I}(\theta \mp \tilde{\theta}, \lambda \mp \widetilde{\lambda})$. These $Y^{I}$ insertions are needed to give the right number of delta functions (when combined with the corresponding $Y^{I}$ 's in $|B\rangle)$. The choice of \pm in (4.6) is required to get a non-vanishing amplitude. Instead of the above arguments, the prescription (4.6) can also be regarded as a definition.

Before turning to explicit calculations based on (4.6), let us make some comments. Since the operator we propose in (4.2) is the analogue of $c_{0}$ in the RNS superstring, one may naturally ask how it relates to the zero mode of the $b$-ghost, in its pure spinor version [11, 38. There is no covariant expression for $b_{0}$ itself. However, in [1] it was shown that there is a covariant expression for a "picture-raised" version of $b_{0}$. Moreover, in 39] it was further shown that even though $b_{0}$ itself is not covariant, the action of $b_{0}$ on $U=\lambda^{\alpha} \Phi_{\alpha}$ can be written covariantly as (modulo BRST exact terms)

$$
b_{0}: \lambda^{\alpha} \Phi_{\alpha} \rightarrow G^{\alpha} \Phi_{\alpha}+H^{\alpha \beta} D_{\alpha} \Phi_{\beta}+K^{\alpha \beta \gamma} D_{\gamma} D_{\beta} \Phi_{\alpha}+L^{\alpha \beta \gamma \delta} D_{\delta} D_{\gamma} D_{\beta} \Phi_{\alpha}
$$

Concentrating on the zero mode vertex operator $U_{2, i+3}$, and using the schematic relations (here we only list the zero mode parts) $H^{\alpha \beta} \sim d d, K^{\alpha \beta \gamma} \sim N d+J d$ and $L^{\alpha \beta \gamma \delta} \sim N N+J N+J J$ [11, 39], we see that the effect of $b_{0}$ is to remove three $\theta$ 's and one $\lambda$ from $U_{2, i+3}$. Thus, it is reasonable to expect that $b_{0}$ maps $U_{2, i+3}$ to $U_{1, i}$ (modulo a constant). If this is indeed true then, at least when acting on these particular states, it is also possible that $b_{0}$ has the inverse action to $c_{0}$, as one would expect. Unfortunately, it is not possible to check this in detail as $H^{\alpha \beta}, K^{\alpha \beta \gamma}$ and $L^{\alpha \beta \gamma \delta}$ have not yet been explicitly determined.

Let us now consider a couple of examples, starting with the absorption of a single massless mode by a D9-brane. The vertex operator for the graviton is of the form $U_{1,1}^{n} h_{m n} \widetilde{U}_{1,1}^{n}, c f$. (2.32). The action of $c_{0} \pm \widetilde{c}_{0}$ in (4.6) turns this vertex operator into $-\mathcal{U}_{2 ; 1}^{h} \mp \mathcal{U}_{1 ; 2}^{h} \equiv-U_{2,4}^{n} \widetilde{U}_{1,1}^{n} h_{m n} \mp U_{1,1}^{n} \widetilde{U}_{2,4}^{n} h_{m n}$ and the scattering amplitude becomes

$$
-\left\langle\mathcal{U}_{2,1}^{h} \mid B\right\rangle_{0} \mp\left\langle\mathcal{U}_{1,2}^{h} \mid B\right\rangle_{0} \propto-T_{9} \int \mathrm{d}^{10} x \operatorname{tr}(h),
$$

which is zero, i.e., the correct result (in order to get a non-zero answer one needs to consider lower dimensional D-branes, in which case one finds $\operatorname{tr}(R \cdot h)$ instead of $\operatorname{tr}(h)$, which is also the known result). Notice that since the vertex operators we are dealing with involve zero modes only, the non-zero modes in the boundary state give no contribution. As another example, the vertex operator for a constant $\mathrm{RR}$ potential [25] is proportional to $U_{1,0} C \widetilde{U}_{1,2}+\widetilde{U}_{1,0} \widetilde{C} U_{1,2}$, cf. (2.38). The action of $c_{0} \pm \widetilde{c}_{0}$ in (4.6) turns this into $\mathcal{U}_{2 ; 1}^{C} \pm \mathcal{U}_{1 ; 2}^{C} \equiv\left[U_{2,3} C \widetilde{U}_{1,2}-\widetilde{U}_{1,0} \widetilde{C} U_{2,5}\right] \pm\left[\widetilde{U}_{2,3} \widetilde{C} U_{1,2}-\right.$ $\left.U_{1,0} C \widetilde{U}_{2,5}\right]$ and the scattering amplitude becomes

$$
\left\langle\mathcal{U}_{2 ; 1}^{C} \mid B\right\rangle_{0} \pm\left\langle\mathcal{U}_{1 ; 2}^{C} \mid B\right\rangle_{0} \propto \pm T_{9} \int \mathrm{d}^{10} x \operatorname{tr}(C-\widetilde{C}) \propto \pm T_{9} \int C_{10}
$$


which again is the correct result. Notice that the \pm , describing brane and antibrane, also works out correctly: from (4.10) follows that brane and anti-brane have opposite RR charges, whereas from (4.9) follows that they have the same tension.

One may also investigate the case with one additional vertex operator insertion. For the scattering involving two vertex operators for the graviton, or $B$-field, this is simple since the integrated vertex operators with zero ghost charge are very similar in both the RNS and pure spinor cases. Indeed, both can be written as

$$
\zeta_{m n} \int \mathrm{d}^{2} z\left(\partial X^{m}+i k_{r} L^{r m}\right)\left(\bar{\partial} X^{n}+i k_{s} L^{s n}\right) e^{i k \cdot X}
$$

the only difference being that $L^{m n}=-\Psi^{m} \Psi^{n}$ in the RNS case, whereas it is $L^{m n}=$ $M^{m n}+N^{m n}$ in the pure spinor case. In the pure spinor case there are also subleading terms in the vertex operator, at higher order in the $\theta$ expansion, but these will not contribute to the scattering amplitude as they give rise to expressions with too many $\theta$ zero modes. Since $L^{m n}$ and $-\Psi^{m} \Psi^{n}$ have the same boundary conditions and the same OPE's with the unintegrated vertex operators for the massless modes (this follows immediately from the Lorentz index structure), the scattering amplitudes will necessarily be the same. Thus, we find agreement with the corresponding RNS results, obtained, e.g., in [40, 41]. Consequently, we also find agreement with the terms in the D-brane effective action extracted from these results, see, e.g., 42.

Above we mostly discussed the scattering of massless modes, but it should also be possible to consider the massive modes. Scattering amplitudes in the presence of a D-brane beyond tree level might also be within reach, using the results in [11.

\subsection{Coupling to a Gauge Field}

In this subsection we incorporate the gauge field living on the world-volume of the D-brane into the previous analysis. For simplicity we consider the case of a D9-brane with a constant gauge field, and will comment on the more general case later. The boundary condition involving $x^{m}$ is of course the same as in the RNS superstring:

$$
\left.\left(\partial_{\tau} x^{m}-f^{m}{ }_{n} \partial_{\sigma} x^{n}\right)\right|_{\tau=0}|B(f)\rangle=0 .
$$

This can also be written as (here $\partial_{ \pm}=\partial_{\tau} \pm \partial_{\sigma}$ )

$$
\left.\left(\partial_{-} x^{m}+R(f)_{n}^{m} \partial_{+} x^{n}\right)\right|_{\tau=0}|B(f)\rangle=0, \quad \text { where } \quad R(f)^{m}=\left(\frac{1-f}{1+f}\right)^{m} .
$$

Since the Lorentz current and the supersymmetry generator are physical quantities, it must be the case that their boundary conditions should agree with the ones in the RNS superstring [6], i.e., one must require

$$
\left(L^{m n}+R(f)_{r}^{m} R(f)^{n}{ }_{s} \widetilde{L}^{r s}\right)|B(f)\rangle=0, \quad\left(q_{\alpha} \mp R(f)_{\alpha}{ }^{\beta} \widetilde{q}_{\beta}\right)|B(f)\rangle=0 .
$$


Here $R(f)_{\alpha}{ }^{\beta}$ should agree with the RNS result 6

$$
R(f)_{\alpha}{ }^{\beta}=\operatorname{det}(1+f)^{-1 / 2} \rightleftarrows\left(-\gamma_{m n} f^{m n}\right)_{\alpha}{ }^{\beta},
$$

where $\rightleftarrows$ is the antisymmetrised exponential

$$
Æ\left(-\gamma_{m n} f^{m n}\right)=\sum_{p=0}^{5} \frac{1}{p !}(-)^{p} \gamma_{m_{1} n_{1} \cdots m_{p} n_{p}} f^{m_{1} n_{1}} \cdots f^{m_{p} n_{p}} .
$$

Note that $R(f)_{\alpha}{ }^{\beta}=R(-f)^{\beta}{ }_{\alpha}$. Furthermore we have the relations

$$
R(-f)=R(f)^{-1}, \quad R(f)^{-1} \gamma^{m} R(f)=\left[\frac{1-f}{1+f}\right]{ }_{n}^{m} \gamma^{n} .
$$

Now, the following boundary conditions

$$
\begin{aligned}
& \left(\theta^{\alpha} \pm \widetilde{\theta}^{\beta} R(-f)_{\beta}{ }^{\alpha}\right)|B(f)\rangle=0, \quad\left(p_{\alpha} \mp R(f)_{\alpha}{ }^{\beta} \widetilde{p}_{\beta}\right)|B(f)\rangle=0 \\
& \left(\lambda^{\alpha} \pm \widetilde{\lambda}^{\beta} R(-f)_{\beta}{ }^{\alpha}\right)|B(f)\rangle=0,
\end{aligned}
$$

are consistent with (4.14) and reduce to the correct boundary conditions when $f_{m n}=$ 0 . Furthermore, they imply that the boundary conditions involving $Q, g$ and $h$ are independent of $f_{m n}$ as they should be, and also that, e.g., the boundary condition involving $\lambda \gamma^{m} \theta$ has the same structure as the one involving $\partial_{-} x^{m}$. Note also that the above boundary condition involving $\lambda^{\alpha}$ and $\widetilde{\lambda}^{\alpha}$ is consistent with the pure spinor conditions because of the relations (4.17). An alternative approach one could follow, instead of using the known boundary conditions from the RNS superstring, is to proceed as in section 3.5 and make an ansatz for the boundary conditions involving $\theta^{\alpha}, p_{\alpha}$ and $\lambda^{\alpha}$. Consistency of the other boundary conditions then leads to the same result as above for $R(f)_{\alpha}{ }^{\beta}$.

The prefactor part of the boundary state involving the non-zero modes is closely analogous to the one in sections 3.3, 3.5. The part involving the modes of $\partial x^{m}$ is

$$
\exp \left(-\sum_{k=1}^{\infty} \frac{1}{k} \alpha_{-k}^{m} R_{m n}(f) \widetilde{\alpha}_{-k}^{n}\right)
$$

whereas the prefactor involving the non-zero modes in the $p \theta$ sector is

$$
\exp \left(\mp \sum_{k=1}^{\infty}\left[p_{\alpha,-k} R_{\beta}^{\alpha}(f) \widetilde{\theta}_{-k}^{\beta}-\theta_{-k}^{\alpha} R_{\alpha}{ }^{\beta}(f) \widetilde{p}_{\beta,-k}\right]\right) .
$$

A convenient basis for calculations is to choose $f$ to be block-diagonal with the $2 \times 2$ blocks along the diagonal proportional to $\left(\begin{array}{cc}0 & f_{i} \\ -f_{i} & 0\end{array}\right)$ with $i=1, \ldots, 5$. In this basis $R^{\alpha}{ }_{\beta}(f)$ takes the form

$$
\frac{\left(1-i f_{1} \sigma_{3}\right)}{\sqrt{1+f_{1}^{2}}} \otimes \cdots \otimes \frac{\left(1-i f_{5} \sigma_{3}\right)}{\sqrt{1+f_{5}^{2}}}
$$


Thus the boundary conditions can be written in the $\mathrm{U}(5)$ notation as (see appendix B for more details on the $\mathrm{U}(5)$ notation for spinors)

$$
\begin{aligned}
& \left(\theta^{ \pm \pm \pm \pm \pm} \pm \frac{\left(1 \mp i f_{1}\right)\left(1 \mp i f_{2}\right)\left(1 \mp i f_{3}\right)\left(1 \mp i f_{4}\right)\left(1 \mp i f_{5}\right)}{\sqrt{1+f_{1}^{2}} \sqrt{1+f_{2}^{2}} \sqrt{1+f_{3}^{2}} \sqrt{1+f_{4}^{2}} \sqrt{1+f_{5}^{2}}} \widetilde{\theta}^{ \pm \pm \pm \pm \pm}\right)|B(f)\rangle \\
& \left(p_{ \pm \pm \pm \pm \pm} \mp \frac{\left(1 \pm i f_{1}\right)\left(1 \pm i f_{2}\right)\left(1 \pm i f_{3}\right)\left(1 \pm i f_{4}\right)\left(1 \pm i f_{5}\right)}{\sqrt{1+f_{1}^{2}} \sqrt{1+f_{2}^{2}} \sqrt{1+f_{3}^{2}} \sqrt{1+f_{4}^{2}} \sqrt{1+f_{5}^{2}}} \widetilde{p}_{ \pm \pm \pm \pm \pm}\right)|B(f)\rangle
\end{aligned}
$$

with exactly the same form for the boundary condition involving $\lambda, \widetilde{\lambda}$ as for the one involving $\theta, \tilde{\theta}$. In this form we again note that the boundary condition is consistent with the pure spinor constraints, as can be seen from the explicit solution of the constraint in the $\mathrm{U}(5)$ basis, $c f$. (2.5) . In the $\mathrm{U}(5)$ basis the part of the prefactor in the boundary state involving the non-zero modes for the ghost fields is particularly simple since it can, e.g., be written using only the conjugate pairs $\left(w_{+}, \lambda^{+}\right),\left(w^{a b}, \lambda_{a b}\right)$ and the right-moving partners.

The zero mode part of the boundary state which satisfies the above boundary conditions is

$$
\begin{aligned}
|B(f)\rangle_{0}= & T_{9} \sqrt{\operatorname{det}(1+f)}\left[U_{2,3}^{\alpha} R(-f)_{\alpha}{ }^{\beta} \widetilde{U}_{1,2 ; \beta} \pm U_{2,4}^{m} R(f)_{m n} \widetilde{U}_{1,1}^{n}+U_{2,5 ; \alpha} R(f)^{\alpha}{ }_{\beta} \widetilde{U}_{1,0}^{\beta}\right. \\
& \left. \pm U_{1,2 \alpha} R(f)^{\alpha}{ }_{\beta} \widetilde{U}_{2,3}^{\beta}+U_{1,1}^{m} R(f)_{m n} \widetilde{U}_{2,4}^{n} \pm U_{1,0}^{\alpha} R(-f)_{\alpha}{ }^{\beta} \widetilde{U}_{2,5 ; \beta}\right]|Z\rangle .
\end{aligned}
$$

As in earlier sections, we have suppressed the $Y^{I}$ 's hidden in $|Z\rangle$. In the present case the $Y^{I}$ 's seemingly have an $f_{m n}$-dependence. However, this $f$-dependence can be absorbed into the $C_{\alpha}^{I}$ and $\widetilde{C}_{\alpha}^{I}$ 's and therefore does not give rise to any additional $f$-dependence when calculating correlation functions or scattering amplitudes. In (4.23) we have included an $f$-dependent overall normalisation factor needed to find agreement with the usual D-brane effective action (see below). It may be possible to understand this normalisation factor from a more careful analysis.

Having determined the dependence on the world-volume gauge field in the boundary state, we can now use the resulting expressions to calculate scattering amplitudes and, for instance, derive the D-brane low-energy effective action. However, before turning to this problem, let us discuss some puzzling aspects not touched upon above. In fact, we have obtained the boundary conditions using the closed string language, but one should also be able to derive these boundary conditions directly in the pure spinor superstring, starting from the pure spinor superstring sigma model (2.3) plus a boundary action. Such an analysis was carried out in 43. In that paper, the resulting boundary conditions were expressed in superfield notation. Consistency requires that the superfields satisfy certain constraint equations. These equations put the superfields on-shell and were also obtained in 44 using the superembedding approach for D-branes. Moreover, it was shown in 44, 43] that these equations have 16 (manifest) linear and also 16 (non-manifest) non-linear supersymmetries and that the superfield equations therefore have to imply the (super) DBI equations of motion. 
To make contact with the boundary conditions above (for a constant gauge field strength) one has to expand the superfield equations in components. It may seem strange that in order to obtain the boundary conditions involving just the gauge field one first needs to "solve" the superfield equations (i.e., determine the component expansion), since these equations also encode the equations of motion for the DBI action. It thus might seem that in order to determine the boundary state (which can then be used to obtain the DBI action) one first has to obtain the equations of motion of the DBI action. However, this is not quite so. Rather, in order to find the boundary conditions for a constant gauge field strength one actually does not need to solve the superfield equations completely, but only determine the first few components in the $\theta$ expansion and the (algebraic) relations between them. This will be further discussed in what follows.

Based on comparison with results in the RNS superstring (see, e.g., [45]) one expects that it should also be possible to write the boundary state in the form $e^{S_{\mathrm{b}}(f)}|B(f=0)\rangle$, where $S_{\mathrm{b}}(f)$ is essentially the boundary action. Naively, one would expect $S_{\mathrm{b}}(f) \propto \int \mathrm{d} \sigma\left(\partial_{\sigma} X^{m} a_{m}(X)+\frac{1}{2} L^{m n} f_{m n}\right)$ since this is the form of $S_{\mathrm{b}}(f)$ in the RNS boundary state (see, e.g., [45]). Here $L_{m n}$ is the boundary value of the RNS Lorentz current and one might think that the only modification one needs to do is to replace the RNS Lorentz current by its pure spinor analogue. However, this naive ansatz does not work (as was observed in 46] in the related context of lightcone GS boundary states [7]). In order to understand how to solve the problem, and also how to make contact with the result in [43], let us focus on the $p \theta$ sector. The naive guess would be that $S_{\mathrm{b}}(f)$ involves $-\frac{1}{2} \int \mathrm{d} \sigma f_{m n}(p \pm \widetilde{p}) \gamma^{m n}(\theta \mp \widetilde{\theta})$, in which case $S_{\mathrm{b}}(f)$ would simply be structurally the same as the vertex operator (2.31). However, let us instead (as in [46]) consider the more general form where $S_{\mathrm{b}}(f)$ is $-\frac{1}{2} \int \mathrm{d} \sigma\left(p_{\alpha} \pm \widetilde{p}_{\alpha}\right)\left(\theta^{\beta} \mp \widetilde{\theta}^{\beta}\right) G_{\alpha}^{\beta}(f)$. This leads to the boundary condition

$$
\left[\left(\theta^{\beta} \pm \widetilde{\theta}^{\beta}\right)+\left(\theta^{\beta} \mp \widetilde{\theta}^{\beta}\right) G_{\alpha}^{\beta}(f)\right]|B(f)\rangle .
$$

By comparison with the previous boundary condition we find agreement provided $G_{\alpha}{ }^{\beta}$ is related to $R_{\alpha}{ }^{\beta}$ via $G=(1-R) /(1+R)$. Using this relation we can determine $G_{\alpha}{ }^{\beta}(f)$ using the known form of $R_{\alpha}{ }^{\beta}(f)$. The explicit form is rather complicated but it is easy to show (e.g., using the block-diagonal basis for $f_{m n}$ to simplify the calculation) that for $\mathrm{D} p$-branes with $p>4, G_{\alpha}{ }^{\beta}(f)$ contains more than just a $\gamma_{(2)}$ part; in particular it also has a $\gamma_{(6)}$ piece, meaning that $S_{\mathrm{b}}(f)$ does not just involve the "Lorentz generators" $(p \pm \widetilde{p}) \gamma^{m n}(\theta \mp \widetilde{\theta})$ as one naively would have expected. Furthermore, $G$ is of course in general not linear in $f_{m n}$.

On the other hand, on general grounds one expects $S_{\mathrm{b}}(f)$ to be simply given by the boundary action constructed in [43]. However, this raises a puzzle since in this action only the $\gamma_{(2)}$ piece occurs. To see this we first note that the boundary action given in [43] is written in terms of the fields $W^{\alpha}$ and $F_{m n}$ (which were defined in [43] and are different from the Yang-Mills ones, given in appendix A). Keeping only the leading order terms in the $\theta$ expansion of $W^{\alpha}$ and $F_{m n}$, which depend non-linearly on $f_{m n}$, and using $W^{\alpha} \propto(\theta \mp \widetilde{\theta})^{\beta} G_{\beta}^{\alpha}(f)$ together with the relation $D_{\alpha} W^{\beta}=\frac{1}{4} F_{m n}(f)\left(\gamma^{m n}\right)_{\alpha}{ }^{\beta}$ (which holds for the leading order terms we consider), 
one finds that the boundary action given in [43] contains a term as above, but where $G_{\alpha}{ }^{\beta}(f)$ only has a $\gamma_{(2)}$ piece. In fact, it is even easier to see that only a $\gamma_{(2)}$ piece occurs in the ghost sector, either from the boundary action in [43] or more directly from the boundary condition [43] $\lambda^{\alpha} \pm \widetilde{\lambda}^{\alpha}=-\frac{1}{4}\left(\lambda^{\beta} \mp \widetilde{\lambda}^{\beta}\right)\left(\gamma_{m n}\right)_{\beta}{ }^{\alpha} F_{m n}(f)$ (there is a similar problem with the $\theta, \tilde{\theta}$ boundary condition). Thus there seems to be a contradiction. However, this is easily resolved by replacing $\left(\gamma_{m n}\right)_{\alpha}{ }^{\beta} F_{m n}(f)$ by a multiple of $G_{\alpha}^{\beta}(f)$ (not affecting any of the conclusions in [43]). In fact, such a replacement is also required to get agreement with the results in [4] where the analogue of $\left(\gamma_{m n}\right)_{\beta}{ }^{\alpha} F_{m n}(f)$ was denoted $h_{\alpha}{ }^{\beta}(f)$ and was explicitly shown to contain more than just a $\gamma_{(2)}$ piece (see also [47]). Thus, after this replacement everything agrees. There is still a slight worry in the ghost sector since, as noted above, the boundary action seems to involve more than just the "Lorentz generators" (i.e., more than just $\gamma_{(2)}$ ). This fact seems to be in conflict with the ghost gauge invariance $w_{\alpha} \rightarrow w_{\alpha}+\left(\gamma^{m} \lambda\right)_{\alpha} \Lambda_{m}$, as the term coupling ghosts and gauge field is not invariant. However ${ }^{10}$, the complete action in [43] is invariant under the ghost gauge transformation provided the above boundary conditions hold. This again amounts to replacing $\left(\gamma^{m n}\right)_{\alpha}{ }^{\beta} F_{m n}(f)$ in [43] with a multiple of our $G_{\alpha}{ }^{\beta}(f)$, which is required for consistency with the pure spinor constraint $(c f$., the discussion at the beginning of this section).

We will now test our proposal (4.23). As in RNS [48, 36, 49, the boundary state can be used to calculate terms in the D-brane effective action. In particular, using (4.23) we will determine the $f$-dependence of the D-brane low-energy effective action. One difference compared to the RNS case is that in the pure spinor calculation only zero modes are involved. This makes the determination of the DBI and WZ parts of the actions very similar. More specifically, using the same method as in [48, 36, 49] we shall next determine the linear coupling of the D-brane to both the graviton and the $\mathrm{RR} C$-field potential, and from the obtained result extract the D-brane effective action.

The linear coupling (in the action) to the graviton $h_{m n}\left(g_{m n}=\delta_{m n}+h_{m n}\right)$ is obtained from ( $c f$. (4.9); see the discussion in the previous subsection)

$$
-\left\langle\mathcal{U}_{1,2}^{h} \mid B(f)\right\rangle_{0} \mp\left\langle\mathcal{U}_{2,1}^{h} \mid B(f)\right\rangle_{0} \propto-T_{9} \int \mathrm{d}^{10} x \operatorname{tr}(h R(f)) \sqrt{\operatorname{det}(1+f)}
$$

from which we deduce, following [48, the DBI part of the D-brane effective action, $-T_{9} \int \mathrm{d}^{10} x \sqrt{\operatorname{det}(g+f)}$. The linear coupling (in the action) to the $\mathrm{RR}$ form fields $C_{p}$ is obtained from ( $c f$. (4.10) $)$

$$
\begin{aligned}
\left\langle\mathcal{U}_{1,2}^{C} \mid B(f)\right\rangle_{0} \pm\left\langle\mathcal{U}_{2,1}^{C} \mid B(f)\right\rangle_{0} & \propto \\
\propto & \pm T_{9} \sqrt{\operatorname{det}(1+f)} \int \mathrm{d}^{10} x \operatorname{tr}(C R(f)-R(f) \widetilde{C}) \\
\propto & \pm T_{9} \int C \wedge e^{f},
\end{aligned}
$$

\footnotetext{
${ }^{10}$ We thank N. Berkovits for discussions on this issue.
} 
which we recognise as the WZ part of the action, $\pm T_{9} \int C \wedge e^{F}$. There are several possible extensions of the above analysis which we shall leave for future work [50].

\section{$5 \quad$ Final Remarks}

In this paper we have studied the pure spinor D-brane boundary state in the simplest setting: a flat spacetime background. Clearly, much work remains to be done and many extensions could be studied in the future. Ideally, one would like to address problems which are inaccessible using standard RNS methods.

As we have discussed at length, the hardest issue in the understanding of the boundary state is its zero mode sector. Our main result for this part of the boundary state is given in (3.24), or in slightly more condensed form in (3.25). Our evidence for this result is that it was constructed in order to satisfy the correct boundary conditions. It further leads to the correct couplings to the bulk supergravity fields (i.e., one-point functions), and also correctly reproduces certain scattering amplitudes involving two vertex operator insertions. These tests did involve some further assumptions beyond just the boundary conditions; in particular in section 4.1 we proposed a prescription for calculating scattering amplitudes in the presence of a D-brane. We have also coupled the boundary state to a gauge field (see section 4.2). The boundary conditions for the gauge field are consistent with the pure spinor constraints and the calculation of scattering amplitudes using the framework discussed in section 4.1 leads to expressions which agree with the known low-energy D-brane effective action for the world-volume gauge field.

One would further like to check that $\langle B|P| B\rangle$ (where $P$ is the propagator) is consistent with the known RNS result. This analysis is hampered by the difficulty of constructing the propagator in the pure spinor superstring. We note that in the RNS superstring the total amplitude vanishes after summing over the various NS and $\mathrm{R}$ sectors. However, a ghost zero mode insertion is needed to get a non-vanishing result within each sector. In the pure spinor case it seems that the amplitude also vanishes, as required for consistency, but not as a result of cancellations between expressions involving all modes, rather as a consequence of the cancellation amongst the zero mode pieces alone.

\section{Acknowledgements}

We would like to thank Troels Harmark and Marcos Mariño for collaboration in 2002 on some of the topics discussed in this paper. We would also like to thank Nathan Berkovits and Pietro Antonio Grassi for discussions and comments. RS would like to thank CECS (Valdivia) for very nice hospitality during the course of this work. RS is supported in part by funds provided by the Fundação para a Ciência e a Tecnologia, under the grants SFRH/BPD/7190/2001 and FEDER-POCTI/FNU/38004/2001. 


\section{A Flat $\mathcal{N}=1, d=10$ Superspace}

The $\mathcal{N}=1, d=10$ superspace coordinates are $\left(x^{m}, \theta^{\alpha}\right)$. The supersymmetry transformations acting on superfields are generated by

$$
\mathcal{Q}_{\alpha}=\frac{\partial}{\partial \theta^{\alpha}}-\frac{1}{2}\left(\gamma^{m} \theta\right)_{\alpha} \frac{\partial}{\partial x^{m}}
$$

satisfying

$$
\left\{\mathcal{Q}_{\alpha}, \mathcal{Q}_{\beta}\right\}=-\gamma_{\alpha \beta}^{m} \frac{\partial}{\partial x^{m}}
$$

The vector field $\frac{\partial}{\partial x^{m}}$ is invariant under the supersymmetry transformations, as is the usual supersymmetric derivative,

$$
D_{\alpha}=\frac{\partial}{\partial \theta^{\alpha}}+\frac{1}{2}\left(\gamma^{m} \theta\right)_{\alpha} \frac{\partial}{\partial x^{m}}
$$

which satisfies,

$$
\left\{D_{\alpha}, D_{\beta}\right\}=\gamma_{\alpha \beta}^{m} \frac{\partial}{\partial x^{m}} \text {. }
$$

To describe $\mathcal{N}=1$ super Yang-Mills in superspace one introduces the superfield potentials $A_{\alpha}(x, \theta), A_{m}(x, \theta)$, and their associated field strengths. Here $A_{m}(x, \theta)=$ $a_{m}(x)+\cdots$, where the field $a_{m}(x)$ is identified with the super Yang-Mills gluon. As this construction is fairly well-known, we shall be brief.

An analysis of the superspace Bianchi identities leads to the following relations between the potentials and their field strengths

$$
\begin{aligned}
& D_{\alpha} A_{\beta}+D_{\beta} A_{\alpha}=\gamma_{\alpha \beta}^{m} A_{m}, \\
& D_{\alpha} A_{m}-\partial_{m} A_{\alpha}=\left(\gamma_{m}\right)_{\alpha \beta} W^{\beta}, \\
& \partial_{m} A_{n}-\partial_{n} A_{m} \equiv F_{m n}=-\frac{1}{8}\left(\gamma_{m n}\right)^{\alpha}{ }_{\beta} D_{\alpha} W^{\beta} .
\end{aligned}
$$

Multiplying the first equation with the gamma matrix $\gamma_{n}^{\alpha \beta}$ yields the relation

$$
A_{n}(x, \theta)=\frac{1}{8} \gamma_{n}^{\alpha \beta} D_{\alpha} A_{\beta}(x, \theta),
$$

which defines $A_{n}$ in terms of $A_{\alpha}$. Note that the gauge invariance $A_{\alpha} \rightarrow A_{\alpha}+D_{\alpha} \Omega$ implies the standard gauge invariance $A_{m} \rightarrow A_{m}+\partial_{m} \Omega$. Multiplying instead with $\gamma_{m_{1} \cdots m_{5}}^{\alpha \beta}$ yields

$$
\gamma_{m_{1} \cdots m_{5}}^{\alpha \beta} D_{\alpha} A_{\beta}=0
$$

which can be shown to imply the super Yang-Mills equations of motion, i.e., this superspace constraint puts the theory on-shell. Multiplying the second equation in (A.5) with $\left(\gamma^{m}\right)^{\eta \alpha}$ yields

$$
W^{\eta}=\frac{1}{10}\left(\gamma^{m}\right)^{\eta \alpha}\left(D_{\alpha} A_{m}-\partial_{m} A_{\alpha}\right)
$$


which together with (A.6) defines $W^{\eta}$ in terms of $A_{\alpha}$. Here $W^{\eta}(x, \theta)=\xi^{\eta}(x)+\cdots$, where $\xi^{\eta}(x)$ is identified with the super Yang-Mills gluino field.

For a super Yang-Mills background containing a gluon field $a_{m}(x)$ with constant field strength $f_{m n}$, and a constant gluino field $\xi^{\alpha}$, one can show that the $\theta$ expansions of the above superfields are

$$
\begin{aligned}
A_{\alpha}(x, \theta) & =\frac{1}{2}\left(\gamma^{m} \theta\right)_{\alpha} a_{m}(x)+\frac{1}{3}\left(\gamma^{m} \theta\right)_{\alpha}\left(\theta \gamma_{m} \xi\right)-\frac{1}{32}\left(\theta \gamma^{n p q} \theta\right)\left(\gamma_{n} \theta\right)_{\alpha} f_{p q} \\
A_{m}(x, \theta) & =a_{m}(x)+\theta \gamma_{m} \xi-\frac{1}{8}\left(\theta \gamma_{m n p} \theta\right) f^{n p} \\
W^{\alpha}(x, \theta) & =\xi^{\alpha}+\frac{1}{4}\left(\theta \gamma^{m n}\right)^{\alpha} f_{m n} \\
F_{m n}(x, \theta) & =f_{m n}
\end{aligned}
$$

\section{B The U(5) Formalism}

It will be occasionally useful to temporarily break $\mathrm{SO}(10)$ to $\mathrm{U}(5) \approx \mathrm{U}(1) \times \mathrm{SU}(5)$. Under this breaking pattern, the vector representation of $\mathrm{SO}(10)$ decomposes as $10 \rightarrow 5 \oplus \overline{5}$. The components of a $\mathrm{SO}(10)$ vector $V^{m}$ are related to the components of the two $\mathrm{U}(5)$ representations $v^{a}, v_{a}$, according to $v^{a}=\frac{1}{2}\left(V^{a}+i V^{a+5}\right)$ for the 5 and $v_{a}=\frac{1}{2}\left(V^{a}-i V^{a+5}\right)$ for the $\overline{5}$; here $a=1, \ldots, 5$. Analogous expressions can be derived for a tensor with an arbitrary number of vector indices. The following representation for the $\mathrm{U}(5)$ components $\left(\gamma^{a}\right)_{\alpha \beta}$ and $\left(\gamma_{a}\right)_{\alpha \beta}$ of the $\mathrm{SO}(10)$ gamma matrices $\Gamma_{\alpha \beta}^{m}$ is useful (see, e.g., [51)

$$
\begin{aligned}
& \left(\gamma^{1}\right)_{\alpha \beta}=-\frac{1+\sigma_{3}}{2} \otimes \sigma_{2} \otimes \sigma_{1} \otimes \sigma_{2} \otimes \sigma_{1}, \\
& \left(\gamma^{2}\right)_{\alpha \beta}=-\sigma_{2} \otimes \frac{1+\sigma_{3}}{2} \otimes \sigma_{1} \otimes \sigma_{2} \otimes \sigma_{1}, \\
& \left(\gamma_{1}\right)_{\alpha \beta}=-\frac{1-\sigma_{3}}{2} \otimes \sigma_{2} \otimes \sigma_{1} \otimes \sigma_{2} \otimes \sigma_{1}, \\
& \left(\gamma^{3}\right)_{\alpha \beta}=-\sigma_{2} \otimes \sigma_{1} \otimes \frac{1+\sigma_{3}}{2} \otimes \sigma_{2} \otimes \sigma_{1}, \\
& \left(\gamma_{2}\right)_{\alpha \beta}=-\sigma_{2} \otimes \frac{1-\sigma_{3}}{2} \otimes \sigma_{1} \otimes \sigma_{2} \otimes \sigma_{1}, \\
& \left(\gamma^{4}\right)_{\alpha \beta}=-\sigma_{2} \otimes \sigma_{1} \otimes \sigma_{2} \otimes \frac{1+\sigma_{3}}{2} \otimes \sigma_{1}, \\
& \left(\gamma^{5}\right)_{\alpha \beta}=-\sigma_{2} \otimes \sigma_{1} \otimes \sigma_{2} \otimes \sigma_{1} \otimes \frac{1+\sigma_{3}}{2}, \\
& \left(\gamma_{3}\right)_{\alpha \beta}=-\sigma_{2} \otimes \sigma_{1} \otimes \frac{1-\sigma_{3}}{2} \otimes \sigma_{2} \otimes \sigma_{1}, \\
& \left(\gamma_{4}\right)_{\alpha \beta}=-\sigma_{2} \otimes \sigma_{1} \otimes \sigma_{2} \otimes \frac{1-\sigma_{3}}{2} \otimes \sigma_{1}, \\
& \left(\gamma_{5}\right)_{\alpha \beta}=-\sigma_{2} \otimes \sigma_{1} \otimes \sigma_{2} \otimes \sigma_{1} \otimes \frac{1-\sigma_{3}}{2} .
\end{aligned}
$$

Indices are raised and lowered with $\epsilon^{\alpha \beta}=-\sigma_{1} \otimes \sigma_{2} \otimes \sigma_{1} \otimes \sigma_{2} \otimes \sigma_{1}$ and its inverse $\epsilon_{\alpha \beta}$, according to the rule $T^{\alpha \beta}=\epsilon^{\alpha \delta} T_{\delta \rho} \epsilon^{\rho \beta}$. The above matrices satisfy $\left\{\gamma^{a}, \gamma_{b}\right\}=\delta_{b}^{a}$. From this result it follows that the corresponding $\Gamma^{m}$ 's satisfy $\left\{\Gamma^{m}, \Gamma^{n}\right\}=2 \eta^{m n}$. The above matrices are $32 \times 32$ dimensional, but we will only consider the restricted action on 16 dimensional Weyl spinors. The restriction of $\Gamma_{\alpha \beta}^{m}$ to this subspace will be denoted by $\gamma_{\alpha \beta}^{m}$.

A spinor of $\mathrm{SO}(10)$ is conveniently represented as the direct product of $5 \mathrm{SO}(2)$ spinors. Denoting the $\mathrm{SO}(2)$ spinor $\left(\begin{array}{l}1 \\ 0\end{array}\right)$ by + and $\left(\begin{array}{l}0 \\ 1\end{array}\right)$ by,$- \mathrm{SO}(10)$ spinors are naturally labelled by a composite index $( \pm, \pm, \pm, \pm, \pm)$, where all 32 possible choices are allowed. The above $\gamma$ matrices act on this basis in the natural way. In this basis a 32 dimensional spinor splits into $16 \oplus \overline{16}$. Spinors with an odd (even) number of +'s are Weyl (anti-Weyl) spinors. A sixteen dimensional spinor $\lambda^{\alpha}$ further decomposes as $16 \rightarrow 1 \oplus 5 \oplus \overline{10}$ (and similarly for the $\overline{16}$ representation). The components with one + will be denoted $\lambda^{a}$ and belong to the 5 representation; the components with three +'s will denoted by $\lambda_{a b}=-\lambda_{b a}$ and belong to the $\overline{10}$ representation. Finally, 
the component with five +'s is the singlet and is denoted $\lambda^{+}$(the difference between the number of +'s and -'s divided by 2 is the U(1) quantum number).

Using the above results one can derive the relations (our conventions differ slightly from those of Berkovits)

$$
\lambda^{\alpha}\left(\gamma^{a}\right)_{\alpha \beta} \lambda^{\beta}=2\left[\lambda^{+} \lambda^{a}-\frac{1}{8} \epsilon^{a b c d e} \lambda_{b c} \lambda_{d e}\right], \quad \lambda^{\alpha}\left(\gamma_{a}\right)_{\alpha \beta} \lambda^{\beta}=2 \lambda_{a b} \lambda^{b},
$$

which gives the parameterisation (2.5). Define $w_{\alpha}$ via

$$
w_{+}=e^{-s}(\partial t+a \partial s), \quad w^{a b}=-2 v^{a b}, \quad w_{a}=0,
$$

where $a$ is a normal ordering constant. One can readily check that $w_{\alpha}$ satisfies the following OPE with $\lambda^{\beta}$

$$
w_{\alpha}(y) \lambda^{\beta}(z) \sim \frac{\delta_{\alpha}^{\beta}}{y-z}-\frac{1}{2} \frac{\left(\gamma_{m}\right)^{\beta+}}{y-z} e^{-s}\left(\gamma^{m}\right)_{\alpha \delta} \lambda^{\delta} .
$$

Note that the OPE's between $w_{+}, \lambda^{+}$and $w^{a b}, \lambda_{a b}$ are the canonical ones. In other words, the second term in (B.4) only contributes if " $\beta=b$ ". Using the above formulæ one can show that

$$
\begin{aligned}
& w_{\alpha} \lambda^{\alpha}=w_{+} \lambda^{+}+w_{a} \lambda^{a}+\frac{1}{2} w_{a b} \lambda^{a b}, \quad w_{\alpha}\left(\gamma^{a b}\right)^{\alpha}{ }_{\beta} \lambda^{\beta}=w^{a b} \lambda^{+}+\frac{1}{2} \epsilon^{a b c d e} w_{c} \lambda_{d e}, \\
& w_{\alpha}\left(\gamma_{a b}\right)^{\alpha}{ }_{\beta} \lambda^{\beta}=-w_{+} \lambda_{a b}-\frac{1}{2} \epsilon_{a b c d e} w^{c d} \lambda^{e} \\
& w_{\alpha}\left(\gamma_{b}^{a}\right)^{\alpha}{ }_{\beta} \lambda^{\beta}=-w_{b} \lambda^{a}-w^{a c} \lambda_{c b}-\frac{1}{2} \delta_{b}^{a}\left(w_{+} \lambda^{+}-w_{c} \lambda^{c}+\frac{1}{2} w^{c d} \lambda_{c d}\right) .
\end{aligned}
$$

Using these results one can write down the expressions for the Lorentz scalar $\partial h=$ $\frac{1}{2} w_{\alpha} \lambda^{\alpha}$ and the Lorentz currents $N_{m n}=\frac{1}{2} w_{\alpha}\left(\gamma_{m n}\right)^{\alpha}{ }_{\beta} \lambda^{\beta}$ as

$$
\begin{aligned}
& \partial h=-\frac{1}{2} u_{a b} v^{a b}+\frac{1}{2} \partial t+\frac{3}{2} \partial s, \quad N^{a b}=-e^{s} v^{a b} \\
& N_{a b}=e^{-s}\left(u_{a c}\left(v^{c d} u_{d b}\right)\right)+\frac{1}{2} e^{-s}\left(u_{a b}\left(v^{c d} u_{c d}\right)\right)-\frac{1}{2} u_{a b}\left(e^{-s}(\partial t+\partial s)\right)+e^{-s} \partial u_{a b} \\
& \tilde{N}_{b}^{a}=v^{a c} u_{c b}+\frac{1}{5} \delta_{b}^{a} v^{c d} u_{c d}, \quad N=\frac{1}{\sqrt{5}}\left(-\frac{5}{4}(\partial t-\partial s)+\frac{1}{4} v^{c d} u_{c d}\right) .
\end{aligned}
$$

Here $N_{b}^{a}$ has been decomposed into the traceless part, $\widetilde{N}^{a}{ }_{b}$, and the trace part, $N$, according to $N_{b}^{a}=\widetilde{N}_{b}^{a}+\frac{1}{\sqrt{5}} \delta_{b}^{a} N$. There are normal ordering ambiguities in the above expressions. More precisely, the terms involving $\partial s$ and $\partial u$ are affected by normal ordering. The ambiguities are fixed by requiring that the OPE's have the right properties. In the above expressions we have indicated the normal ordering prescription by parentheses. The covariant OPE's obtained from the above expressions are given in section 2.1.

Using the above formulæ and $N_{m n} N^{m n}=4\left[N_{a b} N^{a b}+N^{a b} N_{a b}-2 N^{a}{ }_{b} N^{b}{ }_{a}\right]$ one can show that the covariant expression for the stress tensor (2.16) reduces to the expression (2.9) The derivation of this result makes extensive use of the following normal ordering rearrangement rules (here the parentheses indicate the normal ordering prescriptions) 


$$
\begin{aligned}
& (A(B C))-(B(A C))=(([A, B]) C), \\
& ((A B) C)-(B(A C))=(A([C, B]))+(([C, A]) B)+([(A B), C]) .
\end{aligned}
$$

\section{References}

[1] N. Berkovits, "Super-Poincaré covariant quantization of the superstring," JHEP 04 (2000) 018, hep-th/0001035

[2] M. Matone, L. Mazzucato, I. Oda, D. Sorokin and M. Tonin, "The superembedding origin of the Berkovits pure spinor covariant quantization of superstrings," Nucl.

Phys. B639 (2002) 182, hep-th/0206104.

[3] N. Berkovits and D. Z. Marchioro, "Relating the Green-Schwarz and pure spinor formalisms for the superstring," JHEP 0501 (2005) 018, hep-th/0412198.

[4] Y. Aisaka and Y. Kazama, "Origin of pure spinor superstring," hep-th/0502208.

[5] C. G. Callan, Jr., C. Lovelace, C. R. Nappi, and S. A. Yost, "Adding holes and crosscaps to the superstring," Nucl. Phys. B293 (1987) 83.

[6] C. G. Callan, Jr., C. Lovelace, C. R. Nappi, and S. A. Yost, "Loop corrections to superstring equations of motion," Nucl. Phys. B308 (1988) 221.

[7] M. B. Green and M. Gutperle, "Light-cone supersymmetry and D-branes," Nucl. Phys. B476 (1996) 484, hep-th/9604091.

[8] P. Di Vecchia and A. Liccardo, "D-branes in string theory I," NATO Adv. Study Inst. Ser. C. Math. Phys. Sci. 556 (2000) 1, hep-th/9912161.

[9] P. Di Vecchia and A. Liccardo, "D-branes in string theory II," hep-th/9912275.

[10] L. Anguelova and P. A. Grassi, "Super D-branes from BRST symmetry," JHEP 11 (2003) 010, hep-th/0307260.

[11] N. Berkovits, "Multiloop amplitudes and vanishing theorems using the pure spinor formalism for the superstring," JHEP 09 (2004) 047, hep-th/0406055

[12] N. Berkovits, "ICTP lectures on covariant quantization of the superstring," hep-th/0209059

[13] N. Berkovits, "Relating the RNS and pure spinor formalisms for the superstring," JHEP 08 (2001) 026, hep-th/0104247.

[14] N. Berkovits and B. C. Vallilo, "Consistency of super-Poincaré covariant superstring tree amplitudes," JHEP 07 (2000) 015, hep-th/0004171.

[15] N. Berkovits, "Cohomology in the pure spinor formalism for the superstring," JHEP 09 (2000) 046, hep-th/0006003. 
[16] N. Berkovits and O. Chandia, "Lorentz invariance of the pure spinor BRST cohomology for the superstring," Phys. Lett. B514 (2001) 394, hep-th/0105149.

[17] N. Berkovits and P. S. Howe, "Ten-dimensional supergravity constraints from the pure spinor formalism for the superstring," Nucl. Phys. B635 (2002) 75, hep-th/0112160

[18] N. Berkovits and O. Chandia, "Massive superstring vertex operator in $D=10$ superspace," JHEP 08 (2002) 040, hep-th/0204121

[19] W. Siegel, "Classical superstring mechanics," Nucl. Phys. B263 (1986) 93.

[20] M. Chesterman, "Ghost constraints and the covariant quantization of the superparticle in ten dimensions," JHEP 02 (2004) 011, hep-th/0212261.

[21] P. A. Grassi, G. Policastro, P. van Nieuwenhuizen and M. Porrati, "Covariant quantization of superstrings without pure spinor constraints," JHEP 10 (2002) 054, hep-th/0112162

[22] Y. Aisaka and Y. Kazama, "Operator mapping between RNS and extended pure spinor formalisms for superstring," JHEP 08 (2003) 047, hep-th/0305221

[23] P. A. Grassi, G. Policastro, and P. van Nieuwenhuizen, "Superstrings and WZNW models," hep-th/0402122

[24] P. A. Grassi, G. Policastro, and P. van Nieuwenhuizen, "The quantum superstring as a WZNW model," Nucl. Phys. B676 (2004) 43, hep-th/0307056.

[25] L. Cornalba, M. S. Costa, and R. Schiappa, "D-brane dynamics in constant Ramond-Ramond potentials, and noncommutative geometry," hep-th/0209164

[26] P. A. Grassi and L. Tamassia, "Vertex operators for closed superstrings," JHEP 07 (2004) 071, hep-th/0405072.

[27] D. Friedan, E. J. Martinec, and S. H. Shenker, "Conformal invariance, supersymmetry and string theory," Nucl. Phys. B271 (1986) 93.

[28] M. Chesterman, "On the cohomology and inner products of the Berkovits superparticle and superstring," hep-th/0404021

[29] J. Polchinski, "String theory, vol. 2: Superstring theory and beyond,". Cambridge Univ. Pr. (1998).

[30] N. Berkovits, "Quantization of the superstring with manifest U(5) super-Poincaré invariance," Phys. Lett. B457 (1999) 94, hep-th/9902099.

[31] P. A. Grassi and G. Policastro, "Super-Chern-Simons theory as superstring theory," hep-th/0412272.

[32] N. Berkovits, "Covariant quantization of the superparticle using pure spinors," JHEP 09 (2001) 016, hep-th/0105050. 
[33] M. Cederwall, B. E. W. Nilsson, and D. Tsimpis, "The structure of maximally supersymmetric Yang-Mills theory: Constraining higher-order corrections," JHEP 06 (2001) 034, hep-th/0102009.

[34] M. Cederwall, B. E. W. Nilsson, and D. Tsimpis, "Spinorial cohomology and maximally supersymmetric theories," JHEP 02 (2002) 009, hep-th/0110069

[35] S. A. Yost, "Bosonized superstring boundary states and partition functions," Nucl. Phys. B321 (1989) 629.

[36] M. Billo, P. Di Vecchia, M. Frau, A. Lerda, I. Pesando, R. Russo and S. Sciuto, "Microscopic string analysis of the D0-D8 brane system and dual R-R states," Nucl. Phys. B526 (1998) 199, hep-th/9802088.

[37] B. Craps, "D-branes and boundary states in closed string theories," hep-th/0004198

[38] I. Oda and M. Tonin, "On the $b$-antighost in the pure spinor quantization of superstrings," hep-th/0409052.

[39] L. Anguelova, P. A. Grassi, and P. Vanhove, "Covariant one-loop amplitudes in $D=11, "$ hep-th/0408171.

[40] S. S. Gubser, A. Hashimoto, I. R. Klebanov, and J. M. Maldacena, "Gravitational lensing by $p$-branes," Nucl. Phys. B472 (1996) 231, hep-th/9601057.

[41] M. R. Garousi and R. C. Myers, "Superstring scattering from D-branes," Nucl. Phys. B475 (1996) 193, hep-th/9603194

[42] C. P. Bachas, P. Bain, and M. B. Green, "Curvature terms in D-brane actions and their M-theory origin," JHEP 05 (1999) 011, hep-th/9903210.

[43] N. Berkovits and V. Pershin, "Supersymmetric Born-Infeld from the pure spinor formalism of the open superstring," JHEP 01 (2003) 023, hep-th/0205154.

[44] S. F. Kerstan, "Supersymmetric Born-Infeld from the D9-brane," Class. Quant. Grav. 19 (2002) 4525, hep-th/0204225.

[45] K. Hashimoto, "Generalized supersymmetric boundary state," JHEP 04 (2000) 023, hep-th/9909095.

[46] A. A. Tseytlin, "Open superstring partition function in constant gauge field background at finite temperature," Nucl. Phys. B524 (1998) 41, hep-th/9802133

[47] V. Akulov, I. A. Bandos, W. Kummer, and V. Zima, " $D=10$ Dirichlet super-9-brane," Nucl. Phys. B527 (1998) 61, hep-th/9802032.

[48] P. Di Vecchia, M. Frau, I. Pesando, S. Sciuto, A. Lerda and R. Russo, "Classical p-branes from boundary state," Nucl. Phys. B507 (1997) 259, hep-th/9707068.

[49] P. Di Vecchia, M. Frau, A. Lerda, and A. Liccardo, "(F,D $p)$ bound states from the boundary state," Nucl. Phys. B565 (2000) 397, hep-th/9906214. 
[50] R. Schiappa and N. Wyllard, "D-brane boundary states in the pure spinor superstring II." Work in progress.

[51] J. J. Atick and A. Sen, "Covariant one loop fermion emission amplitudes in closed string theories," Nucl. Phys. B293 (1987) 317. 